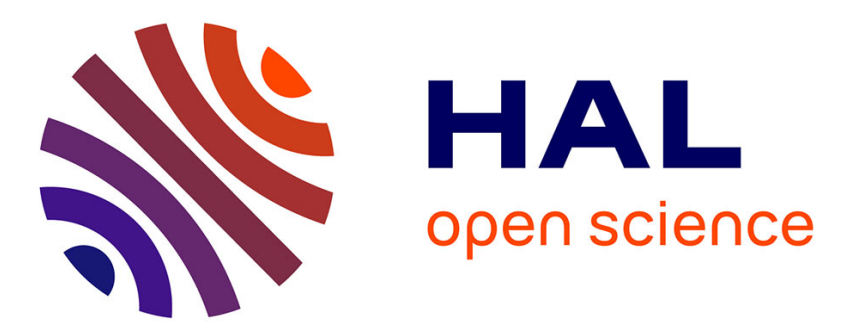

\title{
A splitting method adapted to the simulation of mixed flows in pipes with a compressible two-layer model
}

Charles Demay, Christian Bourdarias, Benoît de Laage de Meux, Stéphane Gerbi, Jean-Marc Hérard

\section{- To cite this version:}

Charles Demay, Christian Bourdarias, Benoît de Laage de Meux, Stéphane Gerbi, Jean-Marc Hérard. A splitting method adapted to the simulation of mixed flows in pipes with a compressible twolayer model. ESAIM: Mathematical Modelling and Numerical Analysis, 2019, 53 (2), pp.405 - 442. 10.1051/m2an/2018051 . hal-01865348

\section{HAL Id: hal-01865348 \\ https://hal.science/hal-01865348}

Submitted on 13 Sep 2018

HAL is a multi-disciplinary open access archive for the deposit and dissemination of scientific research documents, whether they are published or not. The documents may come from teaching and research institutions in France or abroad, or from public or private research centers.
L'archive ouverte pluridisciplinaire HAL, est destinée au dépôt et à la diffusion de documents scientifiques de niveau recherche, publiés ou non, émanant des établissements d'enseignement et de recherche français ou étrangers, des laboratoires publics ou privés. 


\title{
A SPLITTING METHOD ADAPTED TO THE SIMULATION OF MIXED FLOWS IN PIPES WITH A COMPRESSIBLE TWO-LAYER MODEL
}

\author{
Charles Demay ${ }^{1,2}$, Christian Bourdarias ${ }^{2}$, Benoît de Laage de Meux ${ }^{1}$, \\ StéPhane Gerbi ${ }^{2}$ and Jean-Marc HÉrard ${ }^{1,3}$
}

\begin{abstract}
The numerical resolution of the Compressible Two-Layer model proposed in [27] is addressed in this work with the aim of simulating mixed flows and entrapped air pockets in pipes. This five-equation model provides a unified two-phase description of such flows which involve transitions between stratified regimes (air-water herein) and pressurized or dry regimes (pipe full of water or air). In particular, strong interactions between both phases and entrapped air pockets are accounted for. At the discrete level, the coexistence of slow gravity waves in the stratified regime with fast acoustic waves in the pressurized regime is difficult to approximate. Furthermore, the two-phase description requires to deal with vanishing phases in pressurized and dry regimes. In that context, a robust splitting method combined with an implicit-explicit time discretization is derived. The overall strategy relies on the fast pressure relaxation in addition to a mimetic approach with the shallow water equations for the slow dynamics of the water phase. It results in a three-step scheme which ensures the positivity of heights and densities under a CFL condition based on the celerity of material and gravity waves. In that framework, an implicit relaxation-like approach provides stabilization terms which are activated according to the flow regime. Numerical experiments are performed beginning with a Riemann problem for the convective part. The overall approach is then assessed considering relevant mixed flow configurations involving regime transitions, vanishing phases and entrapped air pockets.
\end{abstract}

1991 Mathematics Subject Classification. 35Q35, 65M08, 76T10, 76M12.

January 5, 2018.

\section{INTRODUCTION}

This work is devoted to the simulation of the so-called mixed flows in pipes. The latter are encountered in several industrial areas such as nuclear and hydroelectric power plants or sewage pipelines. They are multiregime flows featuring stratified regimes (air-water in the sequel) and pressurized or dry regimes (pipe full of water or air). In particular, transitions occur between these regimes which potentially result in entrapped air pockets. This transient nature may lead to strong constraints on industrial facilities. For instance, the transitions between stratified and pressurized regimes induce strong pressure variations. In addition, the presence of air,

\footnotetext{
Keywords and phrases: two-layer model, hyperbolic model, mixed flow, splitting method, implicit-explicit scheme.

1 EDF R\&D, dpt. Mécanique des Fluides, Énergetique et Environnement, 6 quai Watier, 78401 Chatou cedex, France. e-mail: charles.demay@edf.fr ; benoit.de-laage-de-meux@edf.fr ; jean-marc.herard@edf.fr.

${ }^{2}$ Laboratoire de Mathématiques, UMR 5127-CNRS and Université Savoie Mont Blanc, 73376 Le Bourget-du-Lac Cedex, France. e-mail: christian.bourdarias@univ-smb.fr ; stephane.gerbi@univ-smb.fr.

${ }^{3}$ Institut de Mathématiques de Marseille, UMR 7373-CNRS, Université Aix-Marseille and Centrale Marseille, 13453 Marseille Cedex, France.
} 
especially entrapped air pockets, is usually unwanted as it may lead to a reduced efficiency, geysering and damages for pumping systems [43]. In a larger extent, these flows are also involved in the interactions between a free surface and a floating structure such as an iceberg, an off-shore wind turbine or a buoy [39]. Consequently, a particular interest is paid to mixed flows through both modelling and experimental studies.

Regarding the modelling of mixed flows, numerous challenges are raised due to the different nature of each regime. Indeed, the stratified regime is driven by slow gravity waves compared to the fast acoustic waves characterizing the pressurized regime. Furthermore, strong interactions between the water phase and the air phase may also be involved, especially in the presence of entrapped air pockets. Modelling studies have been conducted with the aim of proposing a 1D description adapted to the large time and spatial scales involved in industrial facilities. As presented in a recent literature review [12], several approaches have been proposed. They are mainly single-phase models which focus on the transitions between stratified and pressurized regimes neglecting the air phase. Among them, the most natural (and popular) approach relies on a unified description of the different regimes. This approach was introduced in [24] with the so-called Preissmann Slot model. In the latter, the shallow water equations [7,32], usually dedicated to the modelling of free-surface flows, are used for both stratified and pressurized regimes assuming that there is a narrow slot at the top of the pipe. The width of the slot is then calculated to obtain gravity waves with the same celerity as acoustic waves. This technique is validated against experimental data in $[5,14]$ for example. Recently, another unified approach has been proposed in [33] where the shallow water equations are supplemented with a constraint on the water height describing a roof, i.e. the top of the pipe in our framework. With these approaches, sub-atmospheric pressures in the pressurized regime cannot be modeled as they are, by construction, associated with a transition to the stratified regime. These sub-atmospheric pressures may nevertheless be involved in some configurations such as high points of an hydraulic circuit or water hammer phenomena. Thus, more sophisticated models, also based on a unified single-phase description, have been proposed to account for these pressure levels [11,38,50]. Some of them have also been extended to the modelling of entrapped air pockets in simplified configurations [40,49]. Beyond this physical classification, the different nature of each regime and the associated transitions also induce numerical challenges. In the literature, Finite Volume approaches are principally adopted and strategies relying on Godunov-type schemes [38], Roe-type schemes [11,50] or kinetic schemes [10] may be proposed. However, the transition points are often associated with spurious oscillations due to the discrepancy of wave speeds characterizing each regime, see [51] for a related study. Lastly, even if these models with their associated numerical method may provide a satisfactory single-phase description of mixed flows, they are unable to account for strong interactions between the air and water phase in all the regimes. As recently pointed out in a literature review [12], the development of a 1D model which accounts for these interactions is still needed. Some attempts based on two-fluid models have been proposed assuming an incompressible liquid and a compressible gas, see particularly [4,37]. However, the resulting models are not hyperbolic and the management of the pressurized regime is not clearly defined nor the associated transitions.

In this context, a two-phase flow model, namely the Compressible Two-Layer model, has been proposed in [27]. The derivation process relies on a depth averaging of the isentropic Euler set of equations for each phase. It leads to a five-equation system composed by a transport equation on the liquid height in addition to averaged mass and averaged momentum conservation equations for both phases. Thus, this model belongs to the class of two-phase two-pressure models and significant mathematical properties such as hyperbolicity are obtained. This class of model was first introduced for separated flows with depth averaging in [44] and mainly used afterward in a statistical framework for bubbly or granular flows, see $[6,30]$ for instance. The main originality of the Compressible Two-Layer model relies on the closure law of the interfacial pressure which is derived from the hydrostatic constraint applied to the liquid. Therefore, a unified two-phase description of mixed flows is obtained. Indeed, the model is consistent in some sense with the shallow water equations [7,32] for the water phase (stratified regime) while the whole system degenerates formally towards a single-phase isentropic Euler system when one phase vanishes (pressurized or dry regime). From the numerical point of view, several challenges are raised, above all for mixed flows. The convective part of two-fluid two-pressure models is doted with a complex wave structure which makes difficult the Riemann problem to solve regarding Godunov-type 
methods. Furthermore, the whole system includes relaxation source terms which strongly interact with the convective part involving various time scales. When dealing with mixed flows, two additional difficulties may be identified. Firstly, one has to approximate the slow dynamics of the stratified regime (gravity waves) as well as the fast dynamics of the pressurized regime (acoustic waves). Secondly, dealing with a two-phase flow model, one has to handle vanishing phases in pressurized and dry regimes which are known to raise robustness issues.

Some successful solvers for two-fluid two-pressure models are proposed in the literature. They focus on the convective part of the system and they are mainly time-explicit methods such as Godunov-type schemes [3], HLLC-type schemes [42,48] and relaxation schemes $[2,22,23]$ with a particular interest paid to vanishing phases in the latter two references. However, these methods have to comply with the usual Courant-Friedrichs-Lewy (CFL) condition on the time step which involves the celerity of fast acoustic waves. Dealing with the water phase, this celerity is about $1500 \mathrm{~m} . \mathrm{s}^{-1}$ for pure water so that the resulting CFL condition is very constraining and brings too much diffusion to approximate satisfactorily the slow stratified regime. In order to relax this constraint, a possible approach is to derive an implicit-explicit method (IMEX) where the slow dynamics is treated explicitly while the fast dynamics is treated implicitly. This treatment is associated with a well-chosen splitting of the system, which can be a flux splitting or an operator splitting. In this framework, interesting results are obtained for the approximation of low Mach solutions of the Euler system, see [17, 28,36] among others, and a large time-step scheme for a two-fluid two-pressure model is proposed in [16]. Such an implicitexplicit method is derived in [26] as a first attempt for the numerical resolution of the Compressible Two-Layer model. However, it fails to approximate satisfactorily slow gravity waves resulting from a classical dambreak problem and presents a lack of robustness with vanishing phases. Very recently, the numerical resolution of a five-equation model which presents similarities with the Compressible Two-Layer model has been addressed in [29]. The aim of this work is to handle slug flows which may also be interpreted as mixed flows as they involve transitions between stratified and pressurized regimes. A Roe-type explicit scheme is proposed and their strategy to handle the transitions consists in artificially switching from a two-phase to a single-phase description removing gradually coupling terms and setting a zero density value to vanishing phases. Interesting features are obtained regarding slug flow capturing but the accuracy of the explicit approach regarding slow gravity waves is not presented and the artificial management of transitions is questionable.

The numerical resolution of the Compressible Two-Layer model is addressed herein with the aim of simulating mixed flows and entrapped air pockets in pipes. The model and its properties are first recalled in Section 1 as well as its relevance for the two-phase description of mixed flows. An (operator) splitting method associated with an implicit-explicit scheme is then proposed in Section 2. Unlike for the first attempt proposed in [26], the overall strategy relies on the fast pressure relaxation in addition to a mimetic approach with the shallow water equations for the slow dynamics of the water phase. It results in a three-step scheme which ensures the positivity of heights and densities under a CFL condition based on the celerity of material and gravity waves. In particular, an implicit relaxation-like approach provides stabilization terms which are activated according to the flow regime. Furthermore, the robustness of the method allows to solve both phases in pressurized and dry regimes. Numerical experiments are performed in Section 3 beginning with a Riemann problem for the convective part. The overall approach is then assessed considering relevant mixed flow configurations involving regime transitions, vanishing phases and entrapped air pockets. In particular, an experimental validation is proposed.

\section{The Compressible Two-Layer model}

The Compressible Two-Layer model, referred to as the CTL model hereafter, has been introduced in [27] to deal with gas-liquid mixed flows in pipes. The latter involve stratified regimes (see Figure 1.1) as well as pressurized or dry regimes (pipe full of liquid or gas). The governing equations of this model and its main properties are exposed below. In the sequel, we focus on air-water flows but the general approach applies to gas-liquid flows. 


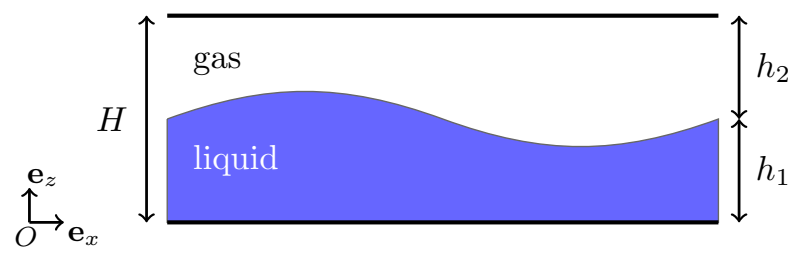

Figure 1.1. Geometric description for horizontal channels.

\subsection{Governing equations}

The CTL model belongs to the class of two-fluid two-pressure models introduced by Ransom \& Hicks in [44]. It results from a depth-averaging of the isentropic Euler set of equations for each phase, see [27] for details. Considering a two-layer air-water flow through an horizontal rectangular pipe of height $H$, see Figure 1.1 , the model reads:

$$
\left\{\begin{array}{l}
\partial_{t} h_{1}+U_{I} \partial_{x} h_{1}=\lambda_{p}\left(P_{I}-P_{2}\left(\rho_{2}\right)\right), \\
\partial_{t} m_{k}+\partial_{x} m_{k} u_{k}=0, k=1,2, \\
\partial_{t} m_{k} u_{k}+\partial_{x} m_{k} u_{k}^{2}+\partial_{x} h_{k} P_{k}\left(\rho_{k}\right)-P_{I} \partial_{x} h_{k}=(-1)^{k} \lambda_{u}\left(u_{1}-u_{2}\right), k=1,2,
\end{array}\right.
$$

where $k=1$ for water, $k=2$ for air, $m_{k}=h_{k} \rho_{k}$ and $h_{1}+h_{2}=H$. Here, $h_{k}, \rho_{k}, P_{k}\left(\rho_{k}\right)$ and $u_{k}$ denote respectively the height, the mean density, the mean pressure and the mean velocity of phase $k$. The interfacial variables, namely the interfacial pressure and the interfacial velocity, are denoted $P_{I}$ and $U_{I}$ respectively. The interface dynamics is represented by the transport equation on $h_{1}$ while the other two equations account for mass and momentum conservation in each phase. Regarding the source terms, $\lambda_{p}$ and $\lambda_{u}$ are positive bounded functions depending on the state variable and accounting for relaxation time scales, they are detailed in Section 1.2 .

The main originality of the CTL model comes from the integration of the hydrostatic constraint applied to the water phase which results in a closure law for $P_{I}$. This constraint is essential in order to account for water gravity waves in the stratified regime. The closure law for the interfacial velocity is obtained using an entropy inequality as in [19]. The resulting closures read:

$$
\left(U_{I}, P_{I}\right)=\left(u_{2}, P_{1}-\rho_{1} g \frac{h_{1}}{2}\right)
$$

where $g$ is the gravity field magnitude. As the phases are compressible, state equations are required for gas and liquid pressures. For instance, perfect gas law may be used for air and linear law for water:

$$
\begin{aligned}
& P_{1}\left(\rho_{1}\right)=\left(\rho_{1}-\rho_{1, \mathrm{ref}}\right) c_{1, \mathrm{ref}}^{2}+P_{1, \mathrm{ref}} \\
& P_{2}\left(\rho_{2}\right)=P_{2, \mathrm{ref}}\left(\frac{\rho_{2}}{\rho_{2, \mathrm{ref}}}\right)^{\gamma_{2}}
\end{aligned}
$$

with some reference density $\rho_{k, \text { ref }}$ and pressure $P_{k, \text { ref. }}$ The celerity of acoustic waves is defined by:

$$
c_{k}=\sqrt{P_{k}^{\prime}\left(\rho_{k}\right)}
$$

where $P_{k}^{\prime}\left(\rho_{k}\right)>0$. For air, $\gamma_{2}$ is set to $7 / 5$ (diatomic gas) and for water, $c_{1}$ is constant and equal to a reference

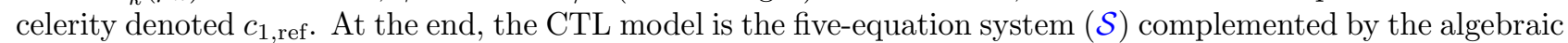
equation $h_{1}+h_{2}=H$, whose six unknowns are $\left(h_{1}, h_{2}, \rho_{1}, \rho_{2}, u_{1}, u_{2}\right)$. 
In the following, the thermodynamic reference state is chosen to deal with air-water flows at $20^{\circ} C: P_{1, \text { ref }}=$ 1.0133 bar, $\rho_{1, \text { ref }}=998.1115 \mathrm{~kg} . \mathrm{m}^{-3}, c_{1, \text { ref }}=1500 \mathrm{~m} . \mathrm{s}^{-1}, P_{2, \text { ref }}=1 \mathrm{~atm}$ and $\rho_{2 \text {,ref }}=1.204 \mathrm{~kg} . \mathrm{m}^{-3}$ which yield $c_{2, \text { ref }} \approx 343 \mathrm{~m} \cdot \mathrm{s}^{-1}$. Note that phase 1 inherits from the fastest pressure waves.

\subsection{Relaxation processes}

The source terms of the CTL model $(\mathcal{S})$ are represented by relaxation terms, namely pressure and velocity relaxation terms. In the following, the associated relaxation processes at the continuous level as well as the relaxation time scales are detailed.

\subsubsection{Pressure relaxation}

The interpretation of the pressure relaxation is given considering a flow homogeneous along $x$. Thus, $(\mathcal{S})$ yields:

$$
\left\{\begin{array}{l}
\partial_{t} h_{1}=\lambda_{p}\left(P_{I}-P_{2}\right), \\
\partial_{t} m_{k}=0, k=1,2 .
\end{array}\right.
$$

The second equation of (1.4) gives $\partial_{t} \rho_{k}=(-1)^{k} \frac{\rho_{k}}{h_{k}} \partial_{t} h_{1}$ such that using the closure law (1.1) for $P_{I}$ and (1.3), one obtains:

$$
\partial_{t}\left(P_{I}-P_{2}\right)=-\left(\frac{c_{1}^{2} \rho_{1}}{h_{1}}+\frac{c_{2}^{2} \rho_{2}}{h_{2}}\right) \lambda_{p}\left(P_{I}-P_{2}\right) .
$$

Therefore, $\Pi(t)=\left(P_{I}-P_{2}\right)(t)$ satisfies $\Pi(t)=\Pi_{0} \exp \left(-\int_{0}^{t} \lambda_{p}\left(\frac{c_{1}^{2} \rho_{1}}{h_{1}}+\frac{c_{2}^{2} \rho_{2}}{h_{2}}\right) d t\right)$ where $\Pi_{0}=\Pi(t=0)$. As $\lambda_{p}$ is a positive bounded function, the following asymptotic behavior is obtained:

$$
P_{I}-P_{2} \underset{t \rightarrow+\infty}{\longrightarrow} 0
$$

and the associated time scale, denoted $\tau_{p}$, is defined as:

$$
\tau_{p}=\left(\lambda_{p}\left(\frac{c_{1}^{2} \rho_{1}}{h_{1}}+\frac{c_{2}^{2} \rho_{2}}{h_{2}}\right)\right)^{-1}
$$

Regarding $\lambda_{p}$, an analytical expression is developed in [31] describing the oscillations of an isolated bubble (air bubble for instance) in an infinite medium (water for instance) with the Rayleigh-Plesset equation. This approach is adopted for our framework so that $\lambda_{p}$ reads:

$$
\lambda_{p}=\frac{3}{4 \pi \mu_{1}} \frac{h_{1} h_{2}}{H},
$$

where $\mu_{1}$ is the dynamic viscosity of phase 1 . For water, $\mu_{1}=10^{-3}$ Pa.s at $T=20^{\circ} \mathrm{C}$. In practice, dealing with air-water flows and choosing $c_{k}=c_{k \text {,ref }}, \rho_{k}=\rho_{k \text {,ref }}, h_{k}=\frac{H}{2}$ with $H=1 m,(1.6)$ yields $\tau_{p} \sim 10^{-12} s$. Consequently, the interfacial pressure $P_{I}$ quickly converges toward the air pressure.

Remark 1.1 (Pressure relaxation with spatial derivatives). When taking into account spatial derivatives in (1.4), one obtains $\Pi(t)=\left(\Pi_{0}+R\right) \exp \left(-\int_{0}^{t} \frac{d \tau}{\tau_{p}}\right)$, where $R=\int_{0}^{t} \mathcal{C} \exp \left(\int_{0}^{\tau} \frac{d \tau^{\prime}}{\tau_{p}}\right) d \tau$ and $\mathcal{C}$ includes spatial derivatives due to convection terms. The relaxation effect still exists with the same time scale $\tau_{p}$ but is in competition with convection effects. Assuming that $\tau_{p}$ is constant and that $\mathcal{C}$ is bounded in time by a constant denoted $M$, one obtains $|R| \leq \tau_{p} M\left(\exp \left(\frac{t}{\tau_{p}}\right)-1\right)$ which provides $\left|R \exp \left(-\int_{0}^{t} \frac{d \tau}{\tau_{p}}\right)\right| \leq|R| \exp \left(-\frac{t}{\tau_{p}}\right) \leq \tau_{p} M\left(1-\exp \left(-\frac{t}{\tau_{p}}\right)\right)$. Thus, the additional contribution due to convection terms vanishes when $\tau_{p} \rightarrow 0$. 


\subsubsection{Velocity relaxation}

As in the previous section, a flow constant along $x$ is considered. Thus, $(\mathcal{S})$ yields:

$$
\left\{\begin{array}{l}
\partial_{t} m_{k}=0, k=1,2 \\
\partial_{t} m_{k} u_{k}=(-1)^{k} \lambda_{u}\left(u_{1}-u_{2}\right), k=1,2
\end{array}\right.
$$

As $m_{k}$ is constant w.r.t. time, $\left(u_{1}-u_{2}\right)(t)$ immediately satisfies:

$$
\partial_{t}\left(u_{1}-u_{2}\right)=-\lambda_{u}\left(\frac{1}{m_{1}}+\frac{1}{m_{2}}\right)\left(u_{1}-u_{2}\right)
$$

which gives $\left(u_{1}-u_{2}\right)(t)=\left(u_{1}-u_{2}\right)(0) \exp \left(-\int_{0}^{t} \lambda_{u}\left(\frac{m_{1}+m_{2}}{m_{1} m_{2}}\right) d t\right)$. As $\lambda_{u}$ is a positive bounded function, the following asymptotic behavior is obtained:

$$
u_{1}-u_{2} \underset{t \rightarrow+\infty}{\longrightarrow} 0
$$

and the associated time scale denoted $\tau_{u}$ verifies:

$$
\tau_{u}=\left(\lambda_{u}\left(\frac{m_{1}+m_{2}}{m_{1} m_{2}}\right)\right)^{-1} .
$$

The function $\lambda_{u}$ is modeled as a classical interfacial drag force which writes:

$$
\lambda_{u}=\frac{1}{2} f_{i} \rho_{2}\left|u_{1}-u_{2}\right|
$$

where $f_{i}$ is a friction factor. In order to define $f_{i}$, several experimental studies have been led since the pioneer work of Taitel and Dukler in 1976 [47]. In particular, $f_{i}$ should ideally depend on the flow regime. In the present work, a constant value relying on experimental results for stratified air-water flows is chosen, that is $f_{i} \sim 0.015$ (see [41]). Indeed, the performed numerical experiments do not involve strong interfacial shear between the phases. However, note that the numerical scheme proposed hereafter is independent of $\lambda_{u}$ so that more complex laws can be implemented.

In practice, dealing with air-water flows and choosing $\rho_{k}=\rho_{k \text {,ref }}, h_{k}=\frac{H}{2}$ with $H=1 \mathrm{~m},(1.10)$ yields $\tau_{u} \sim 7.10^{1} /\left|u_{1}-u_{2}\right|$. With a large speed disparity between the phases, $\left|u_{1}-u_{2}\right| \sim 10$ m.s $s^{-1}$ for instance, one obtains $\tau_{u} \sim 7 \mathrm{~s}$. Therefore, the velocity relaxation time scale is much larger than the pressure relaxation time scale.

\subsection{Relevance of the CTL model for mixed flows}

The CTL model has been developed to deal with mixed flows which involve stratified regimes as well as pressurized or dry regimes (pipe full of water or air). In this section, details are provided regarding its ability to handle these different regimes at the continuous level.

\subsubsection{Consistency with the shallow water equations}

When it comes to free-surface flows, the well-known (incompressible) shallow water equations are usually considered in the literature, see $[1,32]$ for instance. It is thus interesting to check the consistency of the present model with that classical description. Focusing on the momentum conservation equation of $(\mathcal{S})$ for the water phase and smooth solutions, the last two terms of the left hand side, i.e. $\partial_{x} h_{1} P_{1}-P_{I} \partial_{x} h_{1}$, may be rewritten using $P_{I}=P_{1}-\rho_{1} g \frac{h_{1}}{2}$ as:

$$
\partial_{x} h_{1} P_{1}-P_{I} \partial_{x} h_{1}=\partial_{x} h_{1}\left(P_{1}-P_{I}\right)+h_{1} \partial_{x} P_{I}=\partial_{x} \rho_{1} g \frac{h_{1}^{2}}{2}+h_{1} \partial_{x} P_{I}
$$


Thus, the water phase complies with the following system:

$$
\begin{aligned}
& \partial_{t} h_{1}+u_{2} \partial_{x} h_{1}=\lambda_{p}\left(P_{I}-P_{2}\right) \\
& \partial_{t} h_{1} \rho_{1}+\partial_{x} h_{1} \rho_{1} u_{1}=0 \\
& \partial_{t} h_{1} \rho_{1} u_{1}+\partial_{x} h_{1} \rho_{1} u_{1}^{2}+\partial_{x} \rho_{1} g \frac{h_{1}^{2}}{2}+h_{1} \partial_{x} P_{I}=\lambda_{u}\left(u_{2}-u_{1}\right) .
\end{aligned}
$$

If $\rho_{1}$ is considered as a constant in (1.12b) and (1.12c), the convective part reads formally as the classical (incompressible) shallow water model with an hydrostatic gradient and varying interfacial pressure $P_{I}(x, t)$, see [1] for instance. Furthermore, (1.12a) provides the very fast pressure relaxation (1.5) such that the term $h_{1} \partial_{x} P_{I}$ can be read as a source term accounting for varying air pressure. In (1.12), friction effects with the air phase are also taken into account through the velocity relaxation term. The proposed rewriting is relevant in the stratified regime and guides the splitting method developed in Section 2.

\subsubsection{Consistency with pressurized and dry flows}

The case where the pipe is full of phase $k$ is referred to as pressurized flow $(k=1)$ or dry flow $(k=2)$. In practice, transitions from stratified to pressurized or dry regimes often occur in industrial facilities so that one may wonder if this configuration will be correctly handled by the CTL model. Formally, considering $h_{k}=H$, $k=1$ or 2 , with $H$ constant, $(\mathcal{S})$ reduces to:

$$
\left\{\begin{array}{l}
\partial_{t} \rho_{k}+\partial_{x} \rho_{k} u_{k}=0 \\
\partial_{t} \rho_{k} u_{k}+\partial_{x}\left(\rho_{k} u_{k}^{2}+P_{k}\left(\rho_{k}\right)\right)=0,
\end{array}\right.
$$

as soon as the source terms vanish when $h_{k}=H, k=1$ or 2 . This system is equivalent to an isentropic Euler system which is usually used to describe a pipe with constant cross-section and full of one phase including compressibility effects. Consequently, when $h_{k} \rightarrow H, k=1$ or 2 , the CTL model degenerates naturally (actually by construction) towards a relevant model for pressurized and dry flows.

Remark 1.2 (Definition of $\lambda_{u}$ in pressurized and dry regimes). When $h_{k}=H, k=1$ or 2 , the pressure relaxation source term effectively vanishes due to the definition (1.7) of $\lambda_{p}$ while the velocity relaxation source term does not necessarily vanish regarding the definition (1.11) of $\lambda_{u}$. This definition is nonetheless chosen to improve the robustness of the scheme proposed in Section 2 when dealing with vanishing phases. The corresponding parametric study is presented in [25].

Thus, the CTL model provides a unified description of stratified, pressurized and dry regimes. In particular, the water phase is assumed compressible in every regime following a barotropic pressure law. Furthermore, significant mathematical properties are obtained as detailed in the next section.

\subsection{Mathematical properties}

In this section, the main mathematical properties of $(\mathcal{S})$ are recalled. Details and proofs are available in [27].

Property 1.1 (Entropy inequality for $(\mathcal{S})$ ). Smooth solutions of system $(\mathcal{S})$ comply with the entropy inequality:

$$
\partial_{t} \mathcal{E}+\partial_{x} \mathcal{G} \leq 0
$$

where the entropy $\mathcal{E}$ and the entropy flux $\mathcal{G}$ are defined by:

$$
\begin{aligned}
& \mathcal{E}=E_{c, 1}+E_{p, 1}+E_{t, 1}+E_{c, 2}+E_{t, 2}, \\
& \mathcal{G}=u_{1}\left(E_{c, 1}+E_{p, 1}+E_{t, 1}\right)+u_{2}\left(E_{c, 2}+E_{t, 2}\right)+u_{1} h_{1} P_{1}+u_{2} h_{2} P_{2},
\end{aligned}
$$


with:

$$
E_{c, k}=\frac{1}{2} h_{k} \rho_{k} u_{k}^{2}, E_{t, k}=h_{k} \rho_{k} \Psi_{k}\left(\rho_{k}\right), E_{p, 1}=\rho_{1} g \frac{h_{1}^{2}}{2}, \Psi_{k}^{\prime}\left(\rho_{k}\right)=\frac{P_{k}\left(\rho_{k}\right)}{\rho_{k}^{2}} .
$$

Property 1.2 (Hyperbolicity and structure of the convective system). The convective part of $(\mathcal{S})$ is hyperbolic under the non-resonant condition:

$$
\left|u_{1}-u_{2}\right| \neq c_{1} .
$$

Its eigenvalues are unconditionally real and given by:

$$
\lambda_{1}=u_{2}, \lambda_{2}=u_{1}-c_{1}, \lambda_{3}=u_{1}+c_{1}, \lambda_{4}=u_{2}-c_{2}, \lambda_{5}=u_{2}+c_{2} .
$$

The characteristic field associated with the 1-wave $\lambda_{1}$ is linearly degenerate while the characteristic fields associated with the waves $\lambda_{k}, k=2, . ., 5$, are genuinely nonlinear. Moreover, all the Riemann invariants can be detailed.

Note that in our applications, (1.14) should not be violated due to the large acoustic waves celerity in the water phase.

Property 1.3 (Uniqueness of jump conditions for the convective system). Unique jump conditions hold within each isolated field. For all genuinely non-linear fields corresponding to the $k$-waves, $k=2, \ldots, 5$, the RankineHugoniot jump conditions across a single discontinuity of speed $\sigma$ write:

$$
\begin{aligned}
& {\left[h_{k}\right]=0,} \\
& {\left[h_{k} \rho_{k}\left(u_{k}-\sigma\right)\right]=0,} \\
& {\left[h_{k} \rho_{k} u_{k}\left(u_{k}-\sigma\right)+h_{k} P_{k}\right]=0,}
\end{aligned}
$$

where brackets [.] denote the difference between the states on both sides of the discontinuity.

Furthermore, note that as the field associated to the jump of $h_{1}$ is linearly degenerate, the non-conservative products $u_{2} \partial_{x} h_{1}$ and $\left(P_{1}-\rho_{1} g \frac{h_{1}}{2}\right) \partial_{x} h_{1}$ in $(\mathcal{S})$ are well defined. Indeed, one may use the available 1-Riemann invariants to write explicitly the 1 -wave parametrisation. Note that as the jump conditions and the Riemann invariants can be detailed, one can build analytical solutions for the convective part of $(\mathcal{S})$ including the contact discontinuity, shock waves and rarefaction waves. This approach is used in Section 3 to verify the numerical scheme exposed in Section 2.

Property 1.4 (Positivity). Focusing on smooth solutions of $(\mathcal{S})$, the positivity of $h_{k}$ and $\rho_{k}$ is verified, as soon as $\lambda_{p}$ may be written under the form $\lambda_{p}=h_{1} h_{2} \tilde{\lambda}_{p}$, where $\tilde{\lambda}_{p}$ is a positive bounded function depending on the state variable. The positivity property hold for discontinuous solutions of the Riemann problem associated to the convective part of $(\mathcal{S})$.

Due to Property 1.4, the height $h_{k}$ of each layer is naturally kept between the bounds at the continuous level, i.e. $h_{k} \in[0, H], k=1,2$, without imposing any constraint. This is an interesting feature when dealing with mixed flow modelling as this fundamental property may be naturally transposed to the discrete level. For comparison, the popular single-phase mixed flow models available in the literature $[11,38,40,50]$ do not satisfy the constraint $h_{1} \leq H$ so that these models interpret the domain $h_{1} \geq H$ as the pressurized regime. In our case, this property comes from the two-phase framework which nonetheless brings numerical challenges when dealing with vanishing phases. In the next section, a numerical method which handles the different regimes and transitions between them is presented.

\section{A splitting Method ADAPTED TO MiXED FLOWS}

As highlighted in Section 1.3 at the continuous level, the CTL model may be an interesting candidate to compute mixed flows with a two-phase description. Nonetheless, difficulties are encountered at the discrete level 
as listed below. In response, a numerical strategy based on a splitting method combined with an implicit-explicit time discretization is proposed.

\subsection{Numerical challenges}

The CTL model is a two-fluid two-pressure model whose numerical resolution raises several challenges. Firstly, the convective part of the system is doted with a complex wave structure, see Property 1.2, which makes difficult the Riemann problem to solve regarding Godunov-type methods. Secondly, the whole system includes relaxation source terms strongly interacting with the convective part and associated with various time scales. In particular, the pressure relaxation is very fast, see Section 1.2. Dealing with mixed flows, the following challenges are added:

(i) Multi-regime flow. Mixed flows essentially feature two regimes: the stratified and the pressurized regimes. The stratified regime is mainly driven by slow gravity waves in the liquid phase whose typical celerity is $\sqrt{g h_{1}}$ (see [32]), whereas the pressurized regime is driven by fast acoustic waves whose celerity is given by $c_{1}=\sqrt{P_{1}^{\prime}\left(\rho_{1}\right)}$. In practice, $c_{1} \sim 1500 \mathrm{~m} . \mathrm{s}^{-1}$ for water which leads to $\frac{\sqrt{g h_{1}}}{c_{1}} \ll 1$ considering realistic pipe heights. Regarding the characteristic waves of the CTL model, see (1.15), one of them is propagating at slow material speed $u_{2}$ whereas the other four are propagating at fast acoustic speeds, $u_{k} \pm c_{k}, k=1,2$. Therefore, when using classical explicit schemes, one obtains a CFL condition based on the speed of these fast acoustic waves which brings large numerical diffusion in the slow stratified regime. The ideal scheme should be efficient in both regimes in addition to handle transitions between them.

(ii) Vanishing phases in pressurized and dry regimes. At the continuous level, pressurized and dry regimes involve single-phase flows, i.e. $h_{k}=0, k=1$ or 2 . At the discrete level, both phases are solved in every regime with the CTL model. Thus, one has to deal with vanishing phases, i.e. $h_{k} \rightarrow 0, k=1$ or 2 , which raise robustness issues with most of classical numerical solvers.

A numerical scheme is detailed hereafter with the aim of addressing the above challenges. It begins with a splitting approach which particularly accounts for item (i) and for the fast pressure relaxation. A threestep scheme relying on an implicit-explicit time discretization is then derived. As highlighted in the sequel, a particular interest is paid to the robustness of the overall approach with vanishing phases.

\subsection{Operator splitting}

Regarding the CTL model $(\mathcal{S})$, the slow dynamics of the stratified regime is driven by the hydrostatic gradient found in the momentum conservation equation for the water phase while the fast dynamics is driven by pressure gradients in both phases. Therefore, $(\mathcal{S})$ is split into three sub-systems. The slow dynamics of $(\mathcal{S})$ is treated in $\left(\mathcal{S}_{m}\right)$ where the rewriting $(1.12 \mathrm{c})$ is used for the water phase:

$$
\left\{\begin{array}{l}
\partial_{t} h_{1}+u_{2} \partial_{x} h_{1}=\lambda_{p}\left(P_{I}-P_{2}\right) \\
\partial_{t} m_{k}+\partial_{x} m_{k} u_{k}=0, k=1,2 \\
\partial_{t} m_{1} u_{1}+\partial_{x} m_{1} u_{1}^{2}+\partial_{x} \rho_{1} g \frac{h_{1}^{2}}{2}=0 \\
\partial_{t} m_{2} u_{2}+\partial_{x} m_{2} u_{2}^{2}=0 .
\end{array}\right.
$$

The second sub-system $\left(\mathcal{S}_{a}\right)$ refers to the fast dynamics of $(\mathcal{S})$ including the pressure gradients:

$$
\left\{\begin{array}{l}
\partial_{t} h_{1}=0, \\
\partial_{t} m_{k}=0, k=1,2, \\
\partial_{t} m_{1} u_{1}+h_{1} \partial_{x} P_{I}=0 \\
\partial_{t} m_{2} u_{2}+h_{2} \partial_{x} P_{2}+\left(P_{2}-P_{I}\right) \partial_{x} h_{2}=0
\end{array}\right.
$$


where $P_{I}=P_{1}-\rho_{1} g \frac{h_{1}}{2}$. The last sub-system $\left(\mathcal{S}_{u}\right)$ deals with the velocity relaxation source term:

$$
\left\{\begin{array}{l}
\partial_{t} h_{1}=0 \\
\partial_{t} m_{k}=0, k=1,2, \\
\partial_{t} m_{k} u_{k}=(-1)^{k} \lambda_{u}\left(u_{1}-u_{2}\right), k=1,2 .
\end{array}\right.
$$

This splitting has mainly two key features. The first one is the closeness of $\left(\mathcal{S}_{m}\right)$ with the shallow water system for the water phase which is relevant regarding the stratified regime. The second one relies on the resolution of the fast pressure relaxation also in the first step. Doing so, the terms linked to this relaxation, namely $h_{1} \partial_{x} P_{I}$ and $\left(P_{2}-P_{I}\right) \partial_{x} h_{2}$, are explicitly impacted in the second step. Hyperbolicity properties of systems $\left(\mathcal{S}_{m}\right)$ and $\left(\mathcal{S}_{a}\right)$ are given in propositions below. The proofs are not detailed herein as they result from straightforward calculations.

Proposition 2.1 (Structure of the convective part of $\left(\mathcal{S}_{m}\right)$ ). The convective part of $\left(\mathcal{S}_{m}\right)$ is weakly hyperbolic. Its eigenvalues belong to $\left\{u_{2} ; u_{1} \pm \sqrt{g \frac{h_{1}}{2}}\right\}$. The characteristic fields associated with the eigenvalue $u_{2}$ are linearly degenerate while the characteristic fields associated with the eigenvalues $u_{1} \pm \sqrt{g \frac{h_{1}}{2}}$ are genuinely nonlinear.

Proposition 2.2 (Structure of the convective part of $\left(\mathcal{S}_{a}\right)$ ). The convective part of $\left(\mathcal{S}_{m}\right)$ is weakly hyperbolic. Its eigenvalues are $\{0\}$. All the characteristic fields are linearly degenerate.

Remark 2.3 (Eigenvalues and gravity waves). Regarding the water phase equations in $\left(\mathcal{S}_{m}\right)$, one obtains the eigenvalues $u_{1} \pm \sqrt{g \frac{h_{1}}{2}}$ instead of $u_{1} \pm \sqrt{g h_{1}}$ when dealing with the (incompressible) shallow water system. This result is a straightforward consequence of the water phase compressibility which modifies subsequently the Jacobian structure through the hydrostatic gradient $\partial_{x} \rho_{1} g \frac{h_{1}^{2}}{2}$. Indeed, the latter yields contribution in $\partial_{x} \rho_{1}$ in addition to $\partial_{x} h_{1}$ in the compressible framework.

A three step scheme is then proposed and exposed in the next subsections. In particular, a classical explicit scheme with Rusanov fluxes is used for $\left(\mathcal{S}_{m}\right)$ while an original implicit relaxation-like approach is derived to handle the singular spectrum of $\left(\mathcal{S}_{a}\right)$. Note that a similar splitting is developed in [21] for the isentropic Baer-Nunziato system but the underlying scheme is fully explicit.

In the discrete setting, the time step is denoted $\Delta t$ and the space step $\Delta x$. The space is partitioned into cells $C_{i}=\left[x_{i-\frac{1}{2}}, x_{i+\frac{1}{2}}\right.$ [ where $x_{i+\frac{1}{2}}=\left(i+\frac{1}{2}\right) \Delta x$ are the cell interfaces. At discrete times $t^{n}$, the solution is approximated on each cell $C_{i}$ by:

$$
\mathbf{W}_{i}^{n}=\left(\left(h_{1}\right)_{i}^{n},\left(m_{1}\right)_{i}^{n},\left(m_{2}\right)_{i}^{n},\left(m_{1} u_{1}\right)_{i}^{n},\left(m_{2} u_{2}\right)_{i}^{n}\right)^{T} .
$$

\subsection{Step 1: explicit approach for the slow dynamics}

This first step deals with $\left(\mathcal{S}_{m}\right)$ and updates $\mathbf{W}_{i}$ from $\mathbf{W}_{i}^{n}$ to $\mathbf{W}_{i}^{*}$. A classical explicit finite-volume scheme with Rusanov fluxes is used on the convective part while the pressure relaxation source term is treated implicitly except for the $\lambda_{p}$ parameter. It writes:

$$
\mathbf{W}_{i}^{*}=\mathbf{W}_{i}^{n}-\frac{\Delta t}{\Delta x}\left(\mathbf{F}\left(\mathbf{W}_{i+\frac{1}{2}}^{n}\right)-\mathbf{F}\left(\mathbf{W}_{i-\frac{1}{2}}^{n}\right)\right)-\frac{\Delta t}{2 \Delta x} \mathbf{B}\left(\mathbf{W}_{i}^{n}\right)\left(\mathbf{W}_{i+1}^{n}-\mathbf{W}_{i-1}^{n}\right)+\Delta t \mathbf{S}\left(\mathbf{W}_{i}^{*}\right),
$$

where:

$$
\left\{\begin{array}{l}
\mathbf{F}(\mathbf{W})=\left(0, m_{1} u_{1}, m_{2} u_{2}, m_{1} u_{1}^{2}+m_{1} g \frac{h_{1}}{2}, m_{2} u_{2}^{2}\right)^{T} \\
\mathbf{B}(\mathbf{W})=\left(u_{2}, 0,0,0,0\right)^{T} \\
\mathbf{S}(\mathbf{W})=\left(\lambda_{p}\left(P_{I}-P_{2}\right), 0,0,0,0\right)^{T}
\end{array}\right.
$$


The fluxes are defined by:

$$
\left\{\begin{array}{l}
\mathbf{F}\left(\mathbf{W}_{i+\frac{1}{2}}^{n}\right)=\frac{1}{2}\left(\mathbf{F}\left(\mathbf{W}_{i}^{n}\right)+\mathbf{F}\left(\mathbf{W}_{i+1}^{n}\right)-r_{i+\frac{1}{2}}\left(\mathbf{W}_{i+1}^{n}-\mathbf{W}_{i}^{n}\right)\right), \\
r_{i+\frac{1}{2}}=\max _{j \in\{i ; i+1\}}\left(\left|u_{2, j}^{n}\right| ;\left|\left(u_{1} \pm \sqrt{g \frac{h_{1}}{2}}\right)_{j}^{n}\right|\right) .
\end{array}\right.
$$

Note that the time step $\Delta t$ and the coefficient $r_{i+\frac{1}{2}}$ depend on the iteration and should get a superscript $n$ but it is omitted hereafter for the sake of clarity. Regarding the update of $h_{1, i}^{n}$, one can state the following proposition:

Proposition 2.4 (Uniqueness and positivity of heights). There exits a unique $h_{1, i}^{*} \in[0 ; H]$ satisfying the discretization $(2.2)$ of $\left(\mathcal{S}_{m}\right)$. In addition, this property does not require any condition on the time step.

Proof. $h_{1, i}^{*}$ is computed with an implicit treatment of the pressure relaxation source term keeping the relaxation parameter $\lambda_{p}$ explicit. The mass terms $m_{k, i}^{n}$ are updated first and the transport equation (1.12a) is solved under the form $f\left(h_{1, i}^{*}\right)=0$ where:

$$
f(y)=y-h_{1, i}^{n}+\frac{\Delta t}{\Delta x} \int_{x_{i-\frac{1}{2}}}^{x_{i+\frac{1}{2}}} u_{2}^{n} \frac{\partial h_{1}^{n}}{\partial x} d x-\Delta t \lambda_{p, i}^{n}\left(P_{1}\left(\frac{m_{1, i}^{*}}{y}\right)-m_{1, i}^{*} \frac{g}{2}-P_{2}\left(\frac{m_{2, i}^{*}}{H-y}\right)\right) .
$$

One may easily demonstrate that $f$ is strictly increasing on $[0 ; H]$ with the limits $f \rightarrow-\infty$ and $f \rightarrow+\infty$, such that $f(x)=0$ admits a unique solution $h_{1, i}^{*}$ on $[0 ; H]$. This result does not require any condition on $\Delta t$ and is independent of the space discretization applied to $\int_{x_{i-\frac{1}{2}}}^{x_{i+\frac{1}{2}}} u_{2}^{n} \frac{\partial h_{1}^{n}}{\partial x} d x$. In practice, this integral is decomposed as $\int_{x_{i-\frac{1}{2}}}^{x_{i+\frac{1}{2}}} u_{2}^{n} \frac{\partial h_{1}^{n}}{\partial x} d x=\int_{x_{i-\frac{1}{2}}}^{x_{i+\frac{1}{2}}}\left(\frac{\partial u_{2}^{n} h_{1}^{n}}{\partial x}-h_{1}^{n} \frac{\partial u_{2}^{n}}{\partial x}\right) d x$ where the fluxes are defined with (2.3).

Therefore, $h_{1, i}^{*}$ is computed solving $f\left(h_{1, i}^{*}\right)=0$ where $f$ is defined in (2.4). In practice, the Brent method is used to solve this nonlinear problem, see [13]. The algorithm combines linear interpolation and inverse quadratic interpolation with bisection to get efficiency and robustness. The convergence is superlinear and the solution is kept between the bounds, even when getting close to the boundaries. This choice is important when dealing with vanishing phases. Regarding the positivity of densities, it is ensured through a CFL condition detailed in the following proposition:

Proposition 2.5 (Positivity of densities). The scheme for $\left(\mathcal{S}_{m}\right)$ proposed in $(2.2)$ and $(2.3)$ ensures the positivity of densities under the classical CFL condition:

$$
\frac{\Delta t}{\Delta x} \max _{i}\left(\frac{r_{i+\frac{1}{2}}+r_{i-\frac{1}{2}}}{2}\right) \leq 1
$$

which only implies the celerity of material and gravity waves.

Proof. The proof is classical. Applying (2.2) and (2.3) to the mass conservation equations, one readily obtains:

$$
m_{k, i}^{*}=\left(1-\left(r_{i+\frac{1}{2}}+r_{i-\frac{1}{2}}\right) \frac{\Delta t}{2 \Delta x}\right) m_{k, i}^{n}+\left(r_{i+\frac{1}{2}}-u_{k, i+1}^{n}\right) \frac{\Delta t}{2 \Delta x} m_{k, i+1}^{n}+\left(r_{i-\frac{1}{2}}+u_{k, i-1}\right) \frac{\Delta t}{2 \Delta x} m_{k, i-1}^{n}, k=1,2 .
$$

Seeing $m_{k, i}^{*}$ as a linear combination of $m_{k, j}^{n}, j \in\{i-1 ; i ; i+1\}$, with $m_{k, j}^{n} \geq 0$ and $r_{i+\frac{1}{2}} \geq\left|u_{k, l}^{n}\right|, l \in\{i ; i+1\}, \forall i$, a sufficient condition to ensure $m_{k, i}^{*} \geq 0$ is given by (2.5).

The proposed scheme is also consistent with the relaxation property obtained at the continuous level, as presented in the next proposition: 
Proposition 2.6 (Discrete pressure relaxation). The pressure relaxation property exposed in Section 1.2.1 at the continuous level holds at the discrete level. Denoting $\Pi=P_{I}-P_{2}$, it writes $\left|\Pi^{*}\right|<\left|\Pi^{n}\right|$ for a flow homogeneous along $x$.

Proof. Considering a flow independent of space, the semi-discrete equation verified by $h_{k}$ reads:

$$
\frac{\left(h_{1}^{*}-h_{1}^{n}\right)}{\Delta t}=-\frac{\left(h_{2}^{*}-h_{2}^{n}\right)}{\Delta t}=\lambda_{p}^{n} \Pi^{*} .
$$

As $\rho_{k}$ is a continuous and differentiable function of $P_{k}$, one can define two functions $\left(\eta_{1}, \eta_{2}\right)$ such that $\rho_{k}^{*}=$ $\rho_{k}^{n}+\rho_{k}^{\prime}\left(\eta_{k}\right)\left(P_{k}^{*}-P_{k}^{n}\right), k=1,2$ (mean value theorem). With $m_{k}^{*}=m_{k}^{n}$ due to mass conservation without spatial derivatives, one obtains:

$$
\Pi^{*}=\frac{(-1)^{k}}{\lambda_{p}^{n} \Delta t}\left(\frac{m_{k}^{n}}{\rho_{k}^{n}}-\frac{m_{k}^{*}}{\rho_{k}^{*}}\right)=(-1)^{k} \frac{m_{k}^{n}}{\lambda_{p}^{n} \Delta t}\left(\frac{\rho_{k}^{*}-\rho_{k}^{n}}{\rho_{k}^{n} \rho_{k}^{*}}\right)=(-1)^{k} \frac{m_{k}^{n} \rho_{k}^{\prime}\left(\eta_{k}\right)}{\lambda_{p}^{n} \Delta t \rho_{k}^{n} \rho_{k}^{*}}\left(P_{k}^{*}-P_{k}^{n}\right), k=1,2
$$

Using $\Pi^{*}-\Pi^{n}=\left(P_{1}^{*}-P_{1}^{n}\right)+\frac{g}{2}\left(m_{1}^{n}-m_{1}^{*}\right)-\left(P_{2}^{*}-P_{2}^{n}\right)=\left(P_{1}^{*}-P_{1}^{n}\right)-\left(P_{2}^{*}-P_{2}^{n}\right)$, the equation above with $k=1,2$ yields:

which provides:

$$
\Pi^{*}-\Pi^{n}=-\lambda_{p}^{n} \Delta t\left(\frac{\rho_{1}^{*}}{h_{1}^{n} \rho_{1}^{\prime}\left(\eta_{1}\right)}+\frac{\rho_{2}^{*}}{h_{2}^{n} \rho_{2}^{\prime}\left(\eta_{2}\right)}\right) \Pi^{*}
$$

with $\lambda_{p}^{n}>0$ and $\rho_{k}^{\prime}\left(P_{k}\right)>0$.

$$
\left|\Pi^{*}\right|<\left|\Pi^{n}\right|
$$

Remark 2.7 (Discrete pressure relaxation in pressurized and dry regimes). According to the definition (1.7) of $\lambda_{p}$, one observes that $\lambda_{p} \rightarrow 0$ when $h_{k} \rightarrow 0, k=1$ or 2 . In practice, $h_{k}$ does not reach strictly zero and is never set exactly to zero in the initial state such that $\lambda_{p}$ remains strictly positive. Furthermore, using (1.7) for $\lambda_{p}$, one obtains $\tau_{p}=\left(\frac{3}{4 \pi \mu_{1} H}\left(h_{2} c_{1}^{2} \rho_{1}+h_{1} c_{2}^{2} \rho_{2}\right)\right)^{-1}$, where $c_{1}^{2} \rho_{1} \sim 10^{8} \gg 1$ and $c_{2}^{2} \rho_{2} \sim 10^{5} \gg 1$, such that $\tau_{p}>0$ and one may observes that $\tau_{p}<\Delta t$ for reasonable time steps. Thus, in simulated pressurized or dry regimes, pressure relaxation process still exists. This is a positive collateral effect which brings robustness with vanishing phases.

At last, this first step deals with the slow dynamics of $(\mathcal{S})$ ensuring the positivity of heights and densities under the CFL condition (2.5) based on the celerity of material and gravity waves.

\subsection{Step 2: implicit approach for the fast dynamics}

This second step deals with $\left(\mathcal{S}_{a}\right)$ and updates $\mathbf{W}_{i}$ from $\mathbf{W}_{i}^{*}$ to $\mathbf{W}_{i}^{* *}$. In particular, all the variables are kept constant except the velocities. As all the eigenvalues of this system are zero, see proposition (2.2), one cannot apply a classical numerical method relying on the spectral radius of the Jacobian matrix. Thus, a relaxation-like method which consists in considering a larger system easier to solve is developed, see $[2,8,20,21,23]$ for a related framework.

One introduces the system $\left(\mathcal{S}_{a}^{r}\right)$ which relaxes towards $\left(\mathcal{S}_{a}\right)$ in the limit $\epsilon \rightarrow 0$ :

$$
\left\{\begin{array}{l}
\partial_{t} h_{1}=0, \\
\partial_{t} m_{k}=0, k=1,2 \\
\partial_{t} m_{1} u_{1}+h_{1} \partial_{x} \Pi_{I}=0 \\
\partial_{t} m_{2} u_{2}+h_{2} \partial_{x} \Pi_{2}+\left(\Pi_{2}-\Pi_{I}\right) \partial_{x} h_{2}=0 \\
\partial_{t} m_{k} \Pi_{k}+a_{k}^{2} h_{k} \partial_{x} u_{k}+a_{k}^{2}\left(u_{k}-u_{2}\right) \partial_{x} h_{k}=\frac{1}{\epsilon} m_{k}\left(P_{k}-\Pi_{k}\right), k=1,2 .
\end{array}\right.
$$


$\Pi_{k}$ is an additional unknown which relaxes towards $P_{k}$ as $\epsilon \rightarrow 0$ and $\Pi_{I}=\Pi_{1}-\rho_{1} g \frac{h_{1}}{2}$. The PDE verified by $\Pi_{k}$ is derived from the PDE verified by $P_{k}$ in $(\mathcal{S})$ without the convective terms. In addition, $a_{k}$ are positive numerical parameters used to ensure the stability of the relaxation approximation in the regime of small $\epsilon$, their definition is provided later according to the flow regime. The structure of the convective part of $\left(\mathcal{S}_{a}^{r}\right)$ is studied in the following proposition and results from immediate calculations not detailed herein. Note that the positivity of heights and densities is obviously preserved in this step.

Proposition 2.8 (Structure of the convective part of $\left(\mathcal{S}_{a}^{r}\right)$ ). When $a_{k}>0$, the convective part of $\left(\mathcal{S}_{a}^{r}\right)$ is strictly hyperbolic. Its eigenvalues are given by $\left\{0 ; \pm \frac{a_{1}}{\rho_{1}} ; \pm \frac{a_{2}}{\rho_{2}}\right\}$, and all associated characteristic fields are linearly degenerate.

\subsubsection{Time discretization}

In order to keep a CFL condition based on the slow dynamics, the time discretization proposed for the convective part of $\left(\mathcal{S}_{a}^{r}\right)$ is mainly implicit:

$$
\left\{\begin{array}{l}
h_{k}^{* *}=h_{k}^{*}, m_{k}^{* *}=m_{k}^{*}, k=1,2, \\
\left(m_{1}^{* *} u_{1}^{* *}-m_{1}^{*} u_{1}^{*}\right) / \Delta t+h_{1}^{* *} \partial_{x} \Pi_{I}^{* *}=0 \\
\left(m_{2}^{* *} u_{2}^{* *}-m_{2}^{*} u_{2}^{*}\right) / \Delta t+h_{2}^{* *} \partial_{x} \Pi_{2}^{* *}+\left(\Pi_{2}^{*}-\Pi_{I}^{*}\right) \partial_{x} h_{2}^{*}=0, \\
\left(m_{k}^{* *} \Pi_{k}^{* *}-m_{k}^{*} \Pi_{k}^{*}\right) / \Delta t+a_{k}^{2^{*}} h_{k}^{* *} \partial_{x} u_{k}^{* *}+a_{k}^{2^{*}}\left(u_{k}^{*}-u_{2}^{*}\right) \partial_{x} h_{k}^{*}=0, k=1,2 .
\end{array}\right.
$$

The explicit treatment of $\left(\Pi_{2}^{*}-\Pi_{I}^{*}\right) \partial_{x} h_{2}^{*}$ in the air momentum equation is justified by the pressure relaxation solved in the previous step. Regarding the semi-discrete equation verified by $\Pi_{1}$, the term $a_{1}^{2^{*}}\left(u_{1}^{*}-u_{2}^{*}\right) \partial_{x} h_{1}^{*}$ may be treated implicitly solving the air phase first. In practice, this treatment seems needless as the explicit approach does not induce any additional CFL constraint. At last, note that relaxation parameters depend only on $h_{k}$ and $m_{k}$ (see Definition 2.11) leading to $a_{k}^{*}=a_{k}^{* *}$.

In the following, an instantaneous relaxation $(\epsilon \rightarrow 0)$ between $\Pi_{k}$ and $P_{k}$ is assumed and writes $\Pi_{k}^{*}=P_{k}^{*}$. Substituting the equation verified by $\Pi_{k}^{* *}$ into the equations verified by $u_{k}^{* *}$ and dividing by $m_{k}^{*}$, the proposed scheme for $\left(\mathcal{S}_{a}\right)$ reduces to the following semi-discrete equations on $u_{k}, k=1,2$ :

$$
\begin{aligned}
& \frac{u_{1}^{* *}-u_{1}^{*}}{\Delta t}-\frac{\Delta t}{\rho_{1}^{*}} \partial_{x}\left(\frac{a_{1}^{2^{*}}}{\rho_{1}^{*}} \partial_{x} u_{1}^{* *}\right)=-\frac{1}{\rho_{1}^{*}} \partial_{x} P_{I}^{*}+\frac{\Delta t}{\rho_{1}^{*}} \partial_{x}\left(\frac{a_{1}^{2^{*}}\left(u_{1}^{*}-u_{2}^{*}\right)}{m_{1}^{*}} \partial_{x} h_{1}^{*}\right), \\
& \frac{u_{2}^{* *}-u_{2}^{*}}{\Delta t}-\frac{\Delta t}{\rho_{2}^{*}} \partial_{x}\left(\frac{a_{2}^{2^{*}}}{\rho_{2}^{*}} \partial_{x} u_{2}^{* *}\right)=-\frac{1}{\rho_{2}^{*}} \partial_{x} P_{2}^{*}-\frac{\left(P_{2}^{*}-P_{I}^{*}\right)}{m_{2}^{*}} \partial_{x} h_{2}^{*} .
\end{aligned}
$$

One may notice that the additional variables $\Pi_{k}, k=1,2$, do not appear in (2.9). In comparison with $\left(\mathcal{S}_{a}\right)$, the proposed implicit relaxation-like approach acts explicitly as a stabilization process adding diffusion terms weighted by $a_{k}$ at the discrete level. Furthermore, the equation verified by the total momentum may be written under conservative form. Note that the overall approach is referred to as a relaxation-like approach in the sense that a larger system easier to solve is indeed introduced. However, the commonly derived (explicit) relaxation scheme which takes advantage of the linearly degenerate structure of all characteristics fields is not applied here in order to avoid a restricting CFL condition.

\subsubsection{Space discretization}

Considering the space discretization of (2.9), a classical two-point flux approximation is used for the diffusion terms and centered fluxes are used for the pressure gradients of the RHS. Furthermore, in order to improve the robustness of the scheme when $h_{k} \rightarrow 0, k=1,2$, the following is applied in the RHS of (2.9) before space integration:

$$
\frac{\partial_{x} h_{k}^{*}}{m_{k}^{*}}=\frac{1}{\rho_{k}^{*}} \partial_{x} \ln \left(h_{k}^{*}\right),
$$


see [18] for a similar approach. Integrating $(2.9)$ on a cell $C_{i}=\left[x_{i-\frac{1}{2}}, x_{i+\frac{1}{2}}\right.$ [ yields an implicit system for each phase which may be written under matrix form:

$$
A_{k}^{*} \mathbb{U}_{k}^{* *}=\mathbb{S}_{k}^{*}
$$

where $A_{k}^{*}$ is defined as:

$$
A_{k, i j}^{*}= \begin{cases}1+\frac{1}{\rho_{k, i}^{*}}\left(\frac{\Delta t}{\Delta x}\right)^{2}\left(\left(\frac{a_{k}^{2}}{\rho_{k}}\right)_{i+\frac{1}{2}}^{*}+\left(\frac{a_{k}^{2}}{\rho_{k}}\right)_{i-\frac{1}{2}}^{*}\right) & \text { if } i=j, \\ -\frac{1}{\rho_{k, i}^{*}}\left(\frac{\Delta t}{\Delta x}\right)^{2}\left(\frac{a_{k}^{2}}{\rho_{k}}\right)_{i+\frac{1}{2}}^{*} & \text { if } j=i+1, \\ -\frac{1}{\rho_{k, i}^{*}}\left(\frac{\Delta t}{\Delta x}\right)^{2}\left(\frac{a_{k}^{2}}{\rho_{k}}\right)_{i-\frac{1}{2}}^{*} & \text { if } j=i-1, \\ 0 & \text { elsewhere. }\end{cases}
$$

The integrated source terms $\mathbb{S}_{k}^{*}, k=1,2$, write:

$$
\begin{gathered}
\mathbb{S}_{1, i}^{*}=u_{1, i}^{*}-\frac{\Delta t}{2 \Delta x}\left(\frac{P_{I, i+1}^{*}-P_{I, i-1}^{*}}{\rho_{1, i}^{*}}\right)+ \\
\left(\frac{\Delta t}{\Delta x}\right)^{2} \frac{1}{\rho_{1, i}^{*}}\left(\left(\frac{a_{1}^{2}\left(u_{1}-u_{2}\right)}{\rho_{1}}\right)_{i+\frac{1}{2}}^{*} \ln \left(\frac{h_{1, i+1}^{*}}{h_{1, i}^{*}}\right)-\left(\frac{a_{1}^{2}\left(u_{1}-u_{2}\right)}{\rho_{1}}\right)_{i-\frac{1}{2}}^{*} \ln \left(\frac{h_{1, i}^{*}}{h_{1, i-1}^{*}}\right)\right), \\
\mathbb{S}_{2, i}^{*}=u_{2, i}^{*}-\frac{\Delta t}{2 \Delta x}\left(\frac{P_{2, i+1}^{*}-P_{2, i-1}^{*}}{\rho_{2, i}^{*}}\right)-\frac{\Delta t}{\Delta x}\left(\frac{P_{2, i}^{*}-P_{I, i}^{*}}{\rho_{2, i}^{*}}\right) \ln \left(\frac{1+\frac{h_{2, i+1}^{*}}{h_{2, i}^{*}}}{1+\frac{h_{2, i-1}^{*}}{h_{2, i}^{*}}}\right) .
\end{gathered}
$$

Proposition 2.9 (Non-singularity of the implicit system). The system (2.11) is non-singular as $A_{k}^{*}$ has an M-matrix structure.

Proof. Regarding (2.12), $A_{k}^{*}$ verifies:

$$
A_{k, i i}^{*}>0, \quad A_{k, i \neq j}^{*} \leq 0, \quad\left|A_{k, i i}^{*}\right|-\sum_{j \neq i}\left|A_{k, i j}^{*}\right|>0 .
$$

Thus, $A_{k}^{*}$ has an M-matrix structure and (2.11) admits a unique solution.

Remark 2.10 (Non-singularity with vanishing phases). In addition to Proposition 2.9, note that $A_{k}^{*}$ remains non-singular when $h_{k} \rightarrow 0$. This is a substantial property when dealing with pressurized and dry regimes which is not obtained with the implicit system derived in [26].

The definition of the relaxation parameters and the correlated diffusion coefficients is addressed in the next section.

\subsubsection{Definition of relaxation parameters}

At the continuous level, the relaxation parameters $a_{k}$ must typically follow the so-called Whitham condition:

$$
a_{k}>\max _{\rho_{k}}\left(\rho_{k} c_{k}\right), k=1,2,
$$

in order to prevent the relaxation system $\left(\mathcal{S}_{a}^{r}\right)$ from instabilities in the regime of small values of $\epsilon$, see $[9,20]$ for instance. In particular, it ensures the stability of acoustic waves propagating at $\sqrt{P_{k}^{\prime}\left(\rho_{k}\right)}=c_{k}$. As stated throughout this paper, mixed flows involve stratified regimes driven by gravity waves as well as pressurized and dry regimes driven by acoustic waves. Thus, regarding (2.9), a definition of relaxation parameters according to the flow regime is proposed below. 
Definition 2.11 (Relaxation parameters according to the flow regime). Under the light of (2.9), $a_{k}$ is defined according to the flow regime:

- In the stratified and dry regimes $\left(h_{1}<H\right)$ : the pressure gradient $h_{1} \partial_{x} P_{I}$ in $\left(\mathcal{S}_{a}\right)$ is treated as a source term. It accounts for variable interfacial pressure which can be interpreted as air phase pressure due to the fast pressure relaxation solved in the first step. Thus, $a_{1}$ is set to zero in (2.9a).

- In the pressurized regime $\left(h_{1}=H\right)$ : the stabilization process for acoustic waves is applied and $a_{1}$ must follow the so-called Whitham condition: $a_{1}>\max _{\rho_{1}}\left(\rho_{1} c_{1}\right)$ in $(2.9 \mathrm{a})$.

- In all the regimes, $a_{2}$ follows the Whitham condition $a_{2}>\max _{\rho_{2}}\left(\rho_{2} c_{2}\right)$ in $(2.9 \mathrm{~b})$.

According to Definition 2.11, $a_{1}$ switches between the stratified and the pressurized regime from 0 to $\eta_{1} \max _{\rho_{1}}\left(\rho_{1} c_{1}\right)$ where $\eta_{1}$ is a constant greater than one (typically $\eta_{1}=1.01$ ). In practice, it is proposed to identify each regime using a threshold $h_{s}$ on $h_{1}$ so that $a_{1}$ is defined as:

$$
a_{1}=f\left(h_{1}\right) \max _{\rho_{1}}\left(\rho_{1} c_{1}\right)
$$

where:

$$
f\left(h_{1}\right)= \begin{cases}0, & \text { if } h_{1}<h_{s}, \\ \eta_{1}\left(\frac{h_{1}-h_{s}}{H-h_{s}}\right)^{2}, & \text { if } h_{s} \leq h_{1} \leq H .\end{cases}
$$

Therefore, $a_{1}$ is a continuous differentiable function which ensures the stability of (2.9a) in every regime as particularly observed in Section 3. The threshold $h_{s}$ is typically set to $(1-\delta) H$ with $\delta=10^{-3}$ for mixed flow simulations, more details are provided in [25] regarding this setting.

Remark 2.12 (Simplification for constant pipe heights). On the RHS of (2.9a), the diffusion term provided by the relaxation approach writes $\frac{\Delta t}{\rho_{1}^{*}} \partial_{x}\left(\frac{a_{1}^{2^{*}}\left(u_{1}^{*}-u_{2}^{*}\right)}{m_{1}^{*}} \partial_{x} h_{1}^{*}\right)$. In view of Definition 2.11 , this term is zero in the stratified and dry regimes $\left(a_{1}=0\right)$ but also in the pressurized regime when considering constant pipe heights $\left(\partial_{x} h_{1}=0\right)$. This term is thus not considered in the mixed flow simulations presented in Section 3.

At the discrete level, the value $a_{1, i}$ is defined through a simplified version of (2.17) which reads:

$$
a_{1, i}=f\left(h_{1, i}\right) \rho_{1, i} c_{1, i} .
$$

The latter is less restrictive than (2.17) but in our framework, $c_{1}$ is a constant, see (1.2a), and the water density $\rho_{1}$ experiences small variations. An arithmetic mean is then used to define the interfacial diffusion coefficient involved in $A_{1}^{*}$ (see $\left.(2.12)\right)$ :

$$
\left(\frac{a_{1}^{2}}{\rho_{1}}\right)_{i+\frac{1}{2}}^{*}=\frac{1}{2}\left(\frac{a_{1, i}^{2}}{\rho_{1, i}}+\frac{a_{1, i+1}^{2}}{\rho_{1, i+1}}\right)^{*} .
$$

This relation ensures a smoother transition between the regimes compared to the expected harmonic mean. The other interfacial diffusion coefficient arising in the RHS $(2.13)$, i.e. $\left(\frac{a_{1}^{2}\left(u_{1}-u_{2}\right)}{\rho_{1}}\right)_{i+\frac{1}{2}}^{*}$, may be defined equivalently. Regarding the air phase, one directly defines $a_{2, i+\frac{1}{2}}$ in order to be consistent with (2.16) but only between two adjacent cells:

$$
a_{2, i+\frac{1}{2}}=\eta_{2} \max _{j \in\{i, i+1\}}\left(\rho_{2, j} c_{2, j}\right)
$$

where $\eta_{2}$ is a constant greater than one (typically $\eta_{2}=1.01$ ). This discrete definition is more classical when using relaxation schemes, see $[21,23]$ for instance. An arithmetic mean is then used for $\rho_{2, i+\frac{1}{2}}$, leading to the following definition for the interfacial diffusion coefficient involved in $A_{2}^{*}$ (see (2.12)):

$$
\left(\frac{a_{2}^{2}}{\rho_{2}}\right)_{i+\frac{1}{2}}^{*}=a_{2, i+\frac{1}{2}}^{*^{2}}\left(\frac{2}{\rho_{2, i}+\rho_{2, i+1}}\right)^{*} \text {. }
$$


This relation closes the second step which does not require any condition on the time step.

\subsection{Step 3: implicit approach for the velocity relaxation}

This third step deals with $\left(\mathcal{S}_{u}\right)$ and updates $\mathbf{W}_{i}$ from $\mathbf{W}_{i}^{* *}$ to $\mathbf{W}_{i}^{n+1}$. Noticeably, all variables are kept constant except the velocities. As for the pressure relaxation, the source term is treated implicitly except for the $\lambda_{u}$ parameter. Indeed, the latter may include complex functions depending on the state variable and accounting for friction effects, see (1.11). Using the fact that $m_{k}$ is constant w.r.t. time in this step, the proposed semi-discrete implicit scheme writes:

$$
m_{k}^{* *}\left(u_{k}^{n+1}-u_{k}^{* *}\right)=(-1)^{k} \Delta t \lambda_{u}^{* *}\left(u_{1}^{n+1}-u_{2}^{n+1}\right) .
$$

Combining (2.19) for $k=1,2$, one obtains the following non-singular $2 \times 2$ system:

$$
\left(\begin{array}{cc}
m_{1}^{* *}+\Delta t \lambda_{u}^{* *} & -\Delta t \lambda_{u}^{* *} \\
-\Delta t \lambda_{u}^{* *} & m_{2}^{* *}+\Delta t \lambda_{u}^{* *}
\end{array}\right)\left(\begin{array}{c}
u_{1}^{n+1} \\
u_{2}^{n+1}
\end{array}\right)=\left(\begin{array}{c}
\left(m_{1} u_{1}\right)^{* *} \\
\left(m_{2} u_{2}\right)^{* *}
\end{array}\right)
$$

which yields:

$$
\left(\begin{array}{c}
u_{1}^{n+1} \\
u_{2}^{n+1}
\end{array}\right)=\frac{1}{\Lambda^{* *}}\left(\begin{array}{cc}
m_{2}^{* *}+\Delta t \lambda_{u}^{* *} & \Delta t \lambda_{u}^{* *} \\
\Delta t \lambda_{u}^{* *} & m_{1}^{* *}+\Delta t \lambda_{u}^{* *}
\end{array}\right)\left(\begin{array}{c}
\left(m_{1} u_{1}\right)^{* *} \\
\left(m_{2} u_{2}\right)^{* *}
\end{array}\right) .
$$

where $\Lambda^{* *}=m_{1}^{* *} m_{2}^{* *}+\Delta t \lambda_{u}^{* *}\left(m_{1}^{* *}+m_{2}^{* *}\right)$.

Proposition 2.13 (Discrete velocity relaxation). The velocity relaxation property exposed in Section 1.2.2 at the continuous level holds at the discrete level. Denoting $\mathcal{U}^{* *}=u_{1}^{* *}-u_{2}^{* *}$, it writes $\left|\mathcal{U}^{n+1}\right|<\left|\mathcal{U}^{* *}\right|$.

Proof. Using (2.20), one readily obtains:

$$
\mathcal{U}^{n+1}=\frac{m_{1}^{* *} m_{2}^{* *}}{\Lambda^{* *}} \mathcal{U}^{* *}
$$

where $m_{1}^{* *} m_{2}^{* *}<\Lambda^{* *}$.

Remark 2.14 (Discrete velocity relaxation in pressurized and dry regimes). As already mentioned in Remark 1.2 , the chosen definition (1.11) for $\lambda_{u}$, i.e. $\lambda_{u}=\frac{1}{2} f_{i} \rho_{2}\left|u_{1}-u_{2}\right|$, does not vanish when $h_{k} \rightarrow 0$. This definition is relevant at the discrete level as it keeps (2.20) non-singular in pressurized and dry regimes $\left(\Lambda_{u}^{* *}>0\right)$. Note also that this discrete velocity relaxation brings dissipation and thus robustness in these regimes. The corresponding parametric study is presented in [25].

To summarize, the proposed splitting method is a three-step scheme ensuring the positivity of heights and densities under the CFL condition (2.5) based on the celerity of material and gravity waves. In particular, the stable resolution of acoustic waves in the second step is obtained thanks to diffusion terms arising from a relaxation-like method. For the water phase, these terms are activated only in the pressurized regime following a criterion on the water height. Indeed, in the stratified regime, the corresponding convective term to stabilize is treated as a source term accounting for variable air pressure due to the fast pressure relaxation. In the presence of vanishing phases, the overall scheme does not involve any singular system to solve. Furthermore, the dissipative relaxation processes (pressure and velocity) are ensured at the discrete level. In the next section, numerical experiments are performed, beginning with Riemann problems for the convective part and moving to mixed flow configurations.

Remark 2.15 (CFL number and linear stability). In the proposed scheme, the only CFL condition comes from a positivity requirement for heights and densities in the first step. However, stability issues regarding IMEX schemes have been recently pointed out in $[45,52]$. Thus, a linear stability analysis, not presented herein, is detailed in Chapter 5 of [25]. It relies on a Von Neumann approach where each step of the scheme is linearized. In particular, the analysis is performed on a dimensionless version of the CTL model and a critical threshold 
on the CFL number is identified. The latter only involves the ratio $\frac{\sqrt{g H}}{c_{1}}$ and matches remarkably well with numerical experiments performed on the non-linear system. For instance, considering realistic pipe heights satisfying $H \in[0.1 ; 10]$, the maximum CFL number ensuring the linear stability is an increasing function of $\frac{\sqrt{g H}}{c_{1}}$ which belongs to $[0.08 ; 0.7]$, see [25]. In practice, this additional CFL constraint does not yield prohibitive $\mathrm{CPU}$ times as presented in Section 3.

\section{NUMERICAL RESULTS}

In this section, several test cases are performed in order to evaluate the ability of the CTL model associated with the proposed scheme to handle mixed flows. From now on, the proposed scheme is referred to as the SPR scheme for SPlitting with Relaxation. Firstly, one considers in Section 3.1 a Riemann problem to make sure that the SPR scheme is stable and converges towards relevant shock solutions of the homogeneous convective problem. Secondly, the accuracy in the stratified regime is studied in Section 3.2 regarding a dambreak problem. Thirdly, relevant mixed flow configurations are considered including regime transitions, vanishing phases and entrapped air pockets.

\subsection{Shock waves and contact discontinuity: a Riemann problem}

\subsubsection{Global setting and objectives}

This section focuses on the convective part of $(\mathcal{S})$. As stated in section (1.4), the jump conditions and all the Riemann invariants can be detailed so that one can build analytical solutions including the contact discontinuity, shock waves and rarefaction waves. Thus, one considers in the following an analytical solution which involves the five waves of the system: two shocks in each phase propagating at speed $u_{k} \pm c_{k}, k=1,2$, and a contact discontinuity propagating at speed $u_{2}$ where $h_{1}$ jumps, see Figure 3.1.

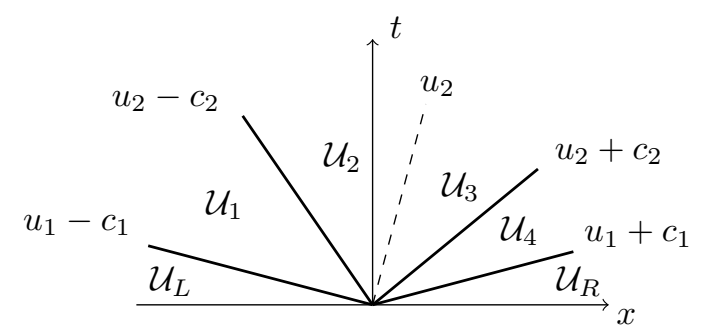

\begin{tabular}{|c|c|c|c|c|c|c|}
\hline Variable & $\mathcal{U}_{L}$ & $\mathcal{U}_{1}$ & $\mathcal{U}_{2}$ & $\mathcal{U}_{3}$ & $\mathcal{U}_{4}$ & $\mathcal{U}_{R}$ \\
\hline$h_{1}$ & 0.5 & 0.5 & 0.5 & 0.5023747 & 0.5023747 & 0.5023747 \\
\hline$\rho_{1}$ & 998.11150 & 998.16140 & 998.16140 & 998.16240 & 998.16240 & 998.06259 \\
\hline$u_{1}$ & 10.0 & 9.9254584 & 9.9254584 & 9.8225555 & 9.82255555 & 9.6734610 \\
\hline$\rho_{2}$ & 1.204 & 1.204 & 1.2642 & 1.2601362 & 1.2349335 & 1.2349335 \\
\hline$u_{2}$ & 5.0 & 5.0 & -11.838960 & -11.838960 & -18.826134 & -18.826134 \\
\hline
\end{tabular}

FIGURE 3.1. Wave structure, initial conditions $\left(\mathcal{U}_{L}, \mathcal{U}_{R}\right)$ and intermediate states $\left(\mathcal{U}_{k}\right)_{k=1,4}$.

As we want to approximate fast acoustic waves (shock waves) in both phases with the SPR scheme, the two relaxation parameters $a_{k}, k=1,2$, follow the Whitham condition. The solutions are computed over the domain $[0,1]$ of the $x$-space where homogeneous Neumann conditions are imposed at both boundaries. The time step is denoted $\Delta t_{\mathrm{m}}$ and is computed from the CFL condition (2.5) where the so-called material CFL number denoted $\mathrm{CFL}_{\mathrm{m}}$ verifies:

$$
\mathrm{CFL}_{\mathrm{m}}=\frac{\Delta t_{\mathrm{m}}}{\Delta x} \max _{i}\left(\frac{r_{\mathrm{m}, i+\frac{1}{2}}+r_{\mathrm{m}, i-\frac{1}{2}}}{2}\right)
$$


with $\mathrm{CFL}_{\mathrm{m}} \leq 1$ and $r_{\mathrm{m}, i+\frac{1}{2}}=\max _{j \in\{i ; i+1\}}\left(\left|u_{2, j}^{n}\right| ;\left|\left(u_{1} \pm \sqrt{g \frac{h_{1}}{2}}\right)_{j}^{n}\right|\right)$ at the $n t h$ iteration.

A mesh refinement is also performed in order to check the numerical convergence of the method. For this purpose, the discrete $L^{1}$-error between the approximate solution and the exact one at the final time $T$, normalized by the discrete $L^{1}$-norm of the exact solution, is computed on regular meshes:

$$
\operatorname{error}_{\mathcal{U}}(\Delta x, T)=\frac{\sum_{j}\left|\mathcal{U}_{j}^{N}-\mathcal{U}_{e x}\left(x_{j}, T\right)\right|}{\sum_{j}\left|\mathcal{U}_{e x}\left(x_{j}, T\right)\right|}
$$

where $\mathcal{U}$ denotes the state vector in non conservative variables, $\mathcal{U}^{N}$ the discrete approximation at final time and $\mathcal{U}_{e x}$ stands for the exact solution. In the refinement process, the coarser mesh is composed of 100 cells and the most refined one contains 200000 cells.

The results obtained with a classical explicit Rusanov scheme applied on the non-split convective part of $(\mathcal{S})$ are added for comparison. The time step of this second scheme is denoted $\Delta t_{\mathrm{a}}$ and is computed from a CFL condition similar to $(2.5)$ which involves the spectral radius of $(\mathcal{S})$ and thus acoustic waves, see Property 1.2. In that framework, the so-called acoustic CFL number is denoted $\mathrm{CFL}_{\mathrm{a}}$ and is defined by:

$$
\mathrm{CFL}_{\mathrm{a}}=\frac{\Delta t_{\mathrm{a}}}{\Delta x} \max _{i}\left(\frac{r_{\mathrm{a}, i+\frac{1}{2}}+r_{\mathrm{a}, i-\frac{1}{2}}}{2}\right),
$$

with $\mathrm{CFL}_{\mathrm{a}} \leq 1$ and $r_{\mathrm{a}, i+\frac{1}{2}}=\max _{j \in\{i ; i+1\}}\left(\left|u_{2, j}^{n}\right| ;\left|\left(u_{1} \pm c_{1}\right)_{j}^{n}\right| ;\left|\left(u_{2} \pm c_{2}\right)_{j}^{n}\right|\right)$ at the $n$th iteration.

For a certain time step computed from a given material $\mathrm{CFL}$ number $\mathrm{CFL}_{\mathrm{m}}$, one can compute the corresponding acoustic CFL number $\mathrm{CFL}_{\mathrm{a}}^{\prime}$ from the relation:

$$
\mathrm{CFL}_{\mathrm{a}}^{\prime}=\frac{\max _{i}\left(\frac{r_{\mathrm{a}, i+\frac{1}{2}}+r_{\mathrm{a}, i-\frac{1}{2}}}{2}\right)}{\max _{i}\left(\frac{r_{\mathrm{m}, i+\frac{1}{2}}+r_{\mathrm{m}, i-\frac{1}{2}}}{2}\right)} \mathrm{CFL}_{\mathrm{m}} .
$$

Focusing on the Riemann problem depicted in Figure 3.1, the SPR scheme is assessed in the sequel setting $\mathrm{CFL}_{\mathrm{m}}=0.5$ and $\mathrm{CFL}_{\mathrm{m}}=0.01$ which corresponds respectively to $\mathrm{CFL}_{\mathrm{a}}^{\prime} \sim 40$ and $\mathrm{CFL}_{\mathrm{a}}^{\prime} \sim 0.8$. The Rusanov scheme is used setting $\mathrm{CFL}_{\mathrm{a}}=0.5$.

Remark 3.1 (SPR scheme with small CFL numbers). When setting a small CFL number with the SPR scheme, typically $\mathrm{CFL}_{\mathrm{a}}^{\prime}=\mathrm{CFL}_{\mathrm{a}}$ which yields $\Delta t_{m}=\Delta t_{a}$, one expects a better resolution of fast propagation phenomena. Furthermore, one also expects a lower numerical diffusion on slow propagation phenomena compared to the full explicit Rusanov scheme as the spectral radius of their explicit part are different. In particular, $r_{m, i+\frac{1}{2}} \ll r_{a, i+\frac{1}{2}}$ when $\left|u_{k}\right| \ll c_{k}$ and $\sqrt{g \frac{h_{1}}{2}} \ll c_{1}$.

\subsubsection{Results and comments}

The fields at $T=23.10^{-5} \mathrm{~s}$ with 1000 cells are displayed on Figure 3.2 and the errors on Figure 3.3. Efficiency results are displayed on Figure 3.4. Despite the great complexity of this test case, one observes that the intermediate states are correctly captured by the two schemes. The latter are stable and converge towards the relevant shock solutions. Note that this property is obtained despite the presence of non-conservative products in $(\mathcal{S}),\left(\mathcal{S}_{m}\right)$ and $\left(\mathcal{S}_{a}\right)$. Indeed, as seen in Property 1.3, these non-conservative products are actually well-defined for shock waves as they involve $\partial_{x} h_{k}$ where $h_{k}$ does not jump across the shocks. The contact discontinuity is also well captured and an expected convergence rate of $\frac{1}{2}$ is obtained, see Figure 3.3.

Focusing on the fastest shock waves, i.e. shock waves within phase 1, the Rusanov scheme is the best suited to approximate them as its CFL condition (3.3) is based on the celerity of these fastest shocks waves $\left(u_{1} \pm c_{1}\right)$. As expected, the SPR scheme with $\mathrm{CFL}_{\mathrm{m}}=0.5$ is the most diffusive one as its CFL condition (3.1) is based 

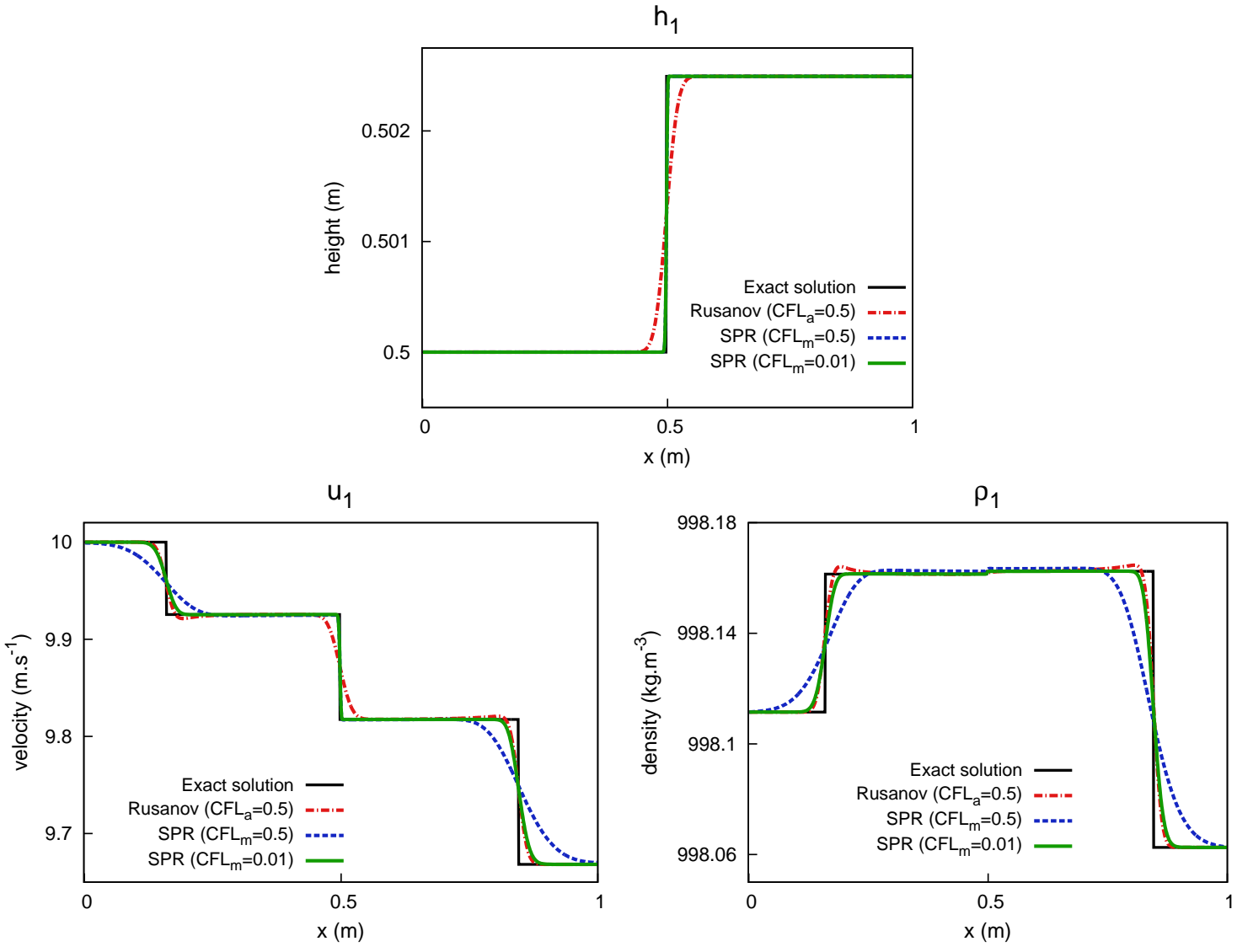

$\mathrm{u}_{2}$
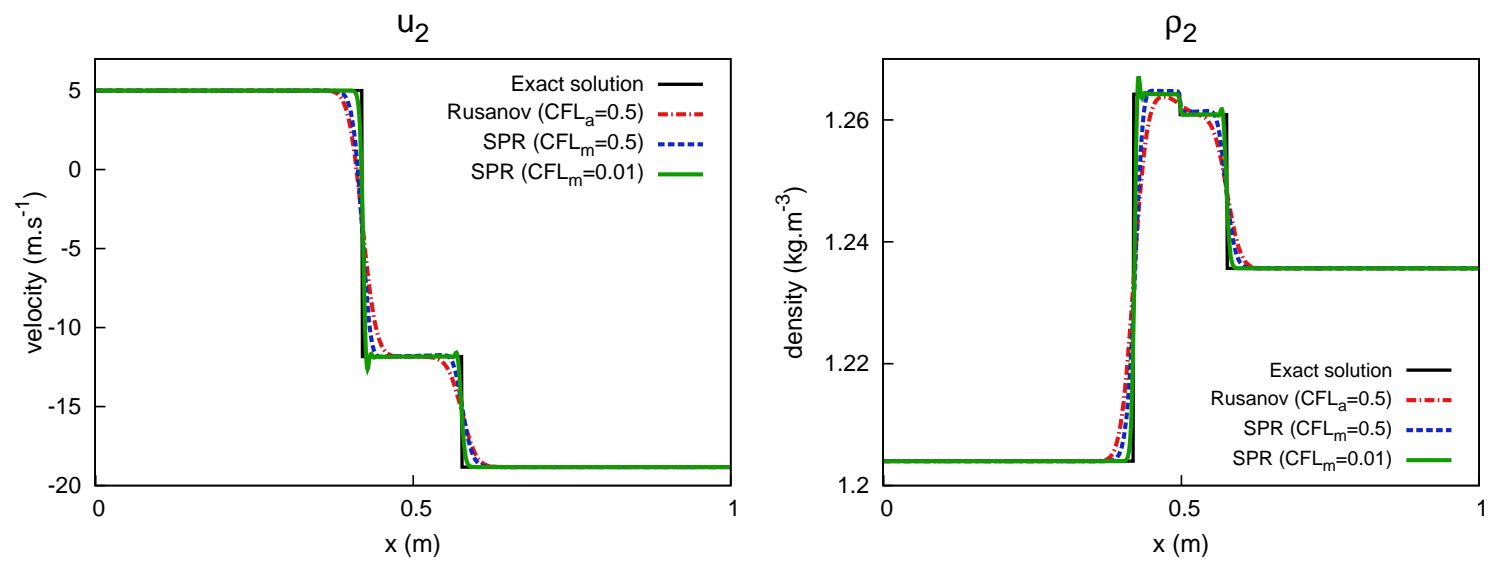

FiguRE 3.2. Approximate solutions for the Riemann problem at $T=23 \cdot 10^{-5} \mathrm{~s}$. with 1000 cells.

on the speed of slow waves. However, setting $\mathrm{CFL}_{\mathrm{m}}=0.01$ improves notably the results which compare well with the Rusanov scheme. These comments are comforted on Figures 3.3 and 3.4 where the SPR scheme with $\mathrm{CFL}_{\mathrm{m}}=0.01$ is equivalent to the Rusanov scheme in terms of errors and efficiency for the variables $\left(\rho_{1}, u_{1}\right)$.

Focusing on the slowest shock waves, i.e. shock waves within phase 2 , the best results are obtained with the SPR scheme setting $\mathrm{CFL}_{\mathrm{m}}=0.01$. Small overshoots are observed on Figure 3.2 but they are bounded in 

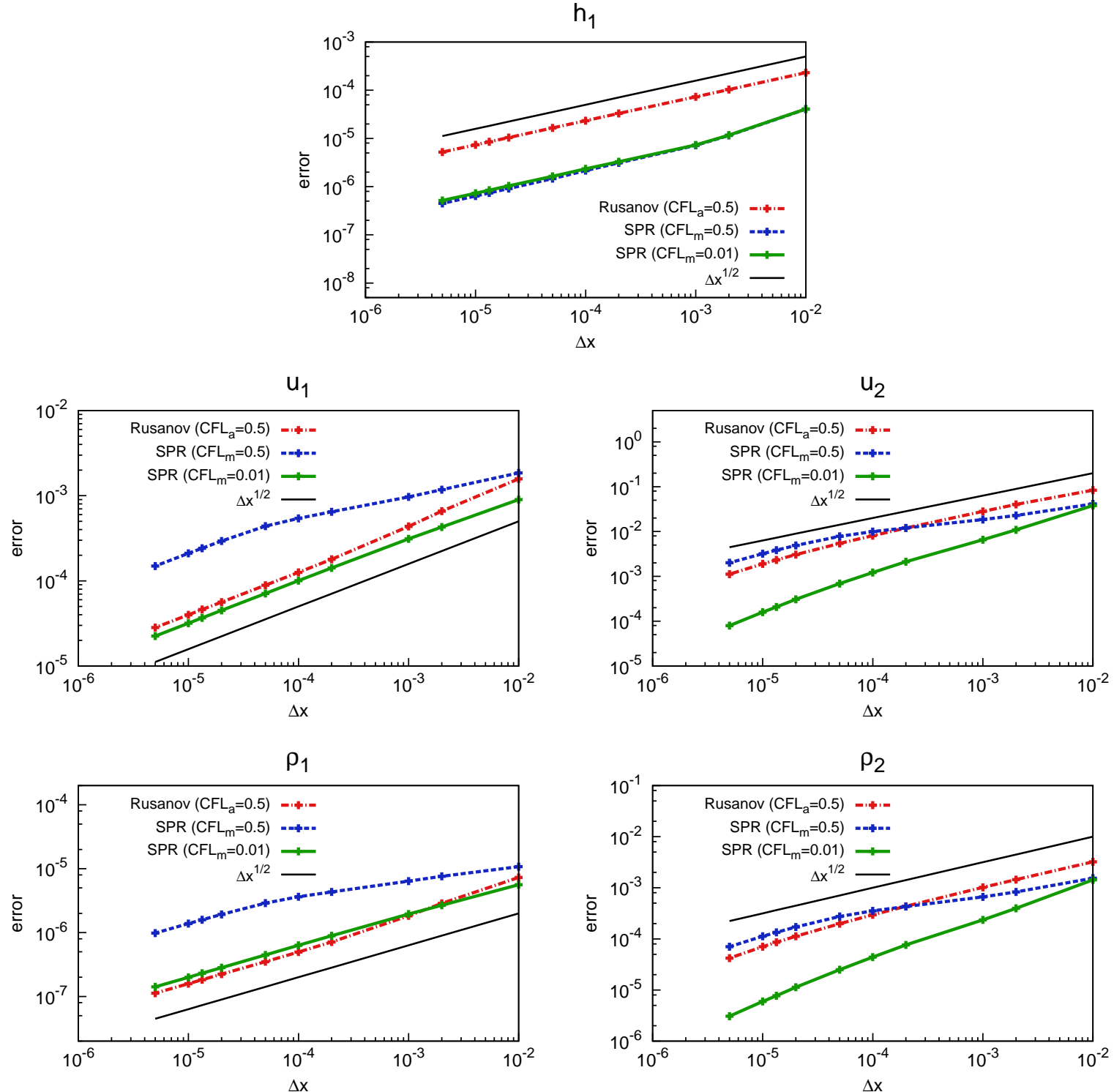

FiguRE 3.3. Errors in $L^{1}$-norm for the Riemann problem.

$L^{1}$-norm and do not preclude the convergence. The Rusanov scheme is the most diffusive and less efficient than the SPR scheme at $\mathrm{CFL}_{\mathrm{m}}=0.5$, see Figure 3.4. Regarding error and efficiency curves, the SPR scheme with $\mathrm{CFL}_{\mathrm{m}}=0.01$ is the best choice for the variables $\left(\rho_{2}, u_{2}\right)$.

Focusing on the slow contact discontinuity where $h_{1}$ jumps, the best results are obtained with the SPR scheme at $\mathrm{CFL}_{\mathrm{m}}=0.5$. Indeed, the latter follows the best suited CFL condition for slow propagation phenomena. As expected, the full explicit Rusanov scheme is not adapted for capturing slow waves and provides the most diffusive results with the worst efficiency, see Figure 3.4. The SPR scheme with $\mathrm{CFL}_{\mathrm{m}}=0.01$ and $\mathrm{CFL}_{\mathrm{m}}=0.5$ compares well in terms of errors, see Figure 3.3, but the second setting is more efficient as larger time steps are used. 

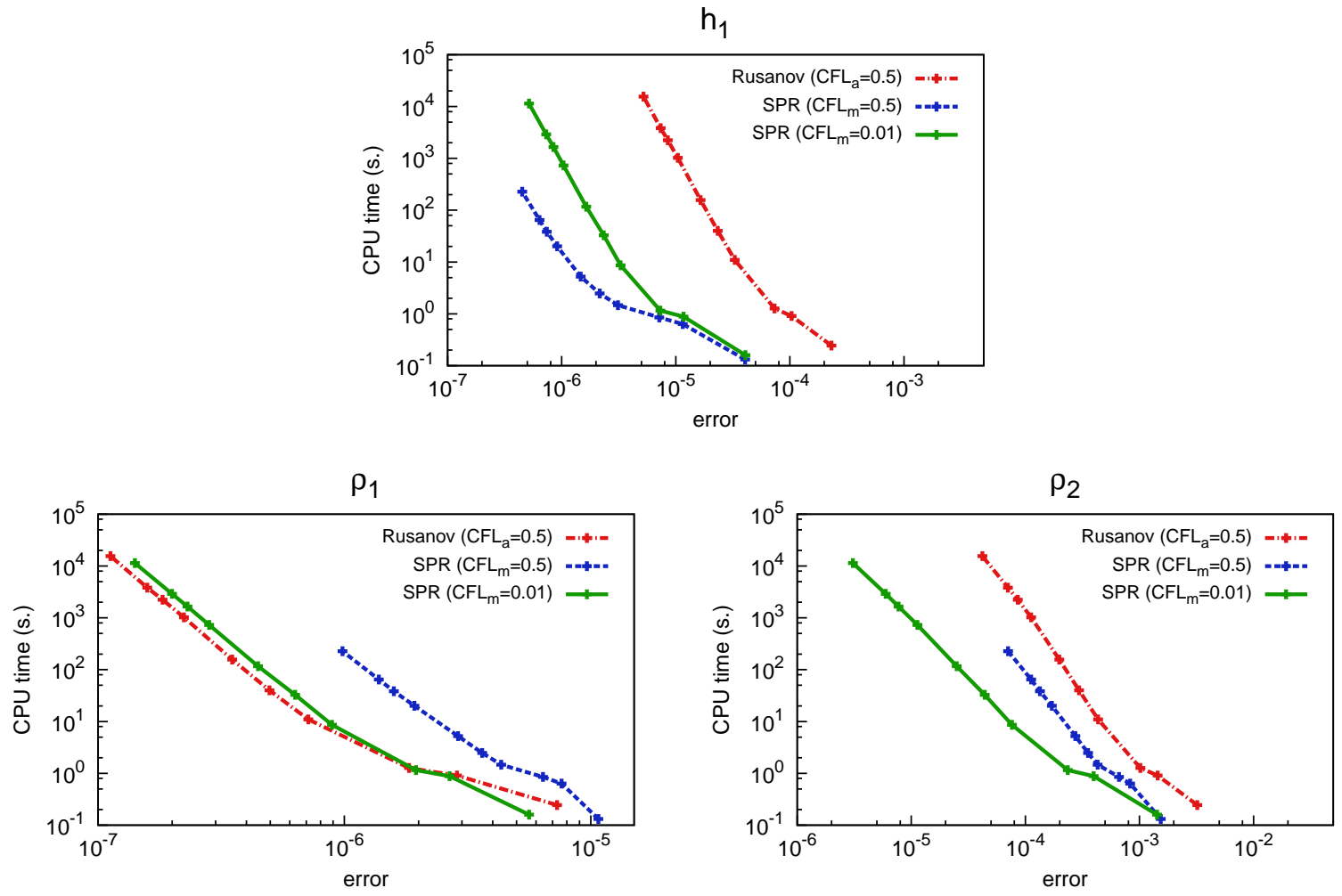

FiguRE 3.4. Errors in $L^{1}$-norm against CPU time for the Riemann problem.

In summary, the SPR scheme is a convergent and stable scheme for the convective part of $(\mathcal{S})$. It yields better accuracy and efficiency compared to a classical explicit Rusanov scheme, in particular when setting $\mathrm{CFL}_{\mathrm{m}}=0.01$. In the next section, these conclusions are confronted to a dambreak test case which involves the full system with pressure and velocity relaxation source terms.

\subsection{Stratified regime: a dambreak problem}

A common way to deal with free-surface flows is to use the well-known Saint-Venant or shallow water equations, see [32]. In a few words, this model is a one-layer model resulting from a depth averaging of the Euler set of equations and assuming a thin layer of incompressible fluid with hydrostatic pressure law. Particularly, it admits an analytical solution for the so-called dambreak problem without friction effects. As detailed below, this configuration is relevant regarding stratified regimes. Thus, it is proposed to consider the dambreak test case for the CTL model and to compare the results with the reference solution provided by the shallow water equations. Indeed, one can expect to obtain the same kind of solution for the water layer as the derivation processes are very close and the compressibility of water as well as the additional air layer are expected to have a minor influence here.

\subsubsection{Global setting and objectives}

The initial condition for the dambreak problem is a discontinuity on $h_{1}$ with uniform density and zero speed, see Figure 3.5. In order to get meaningful results in a short simulation time, hereafter $T=24 \cdot 10^{-2} s$, a pipe of height $H=10 \mathrm{~m}$ and length $L=1 \mathrm{~m}$ is considered (the same trends are obtained with smaller pipes). This configuration provides a low speed flow which is representative of practical configurations with $\frac{\left|u_{1}\right|}{c_{1}} \sim 10^{-3}$ and 
$\frac{\sqrt{g H}}{c_{1}} \sim 7.10^{-3}$. The solutions are computed over the domain $[0,1]$ of the $x$-space with a regular mesh composed of 1000 cells. Wall boundary conditions (mirror states) are imposed at the inlet and outlet.

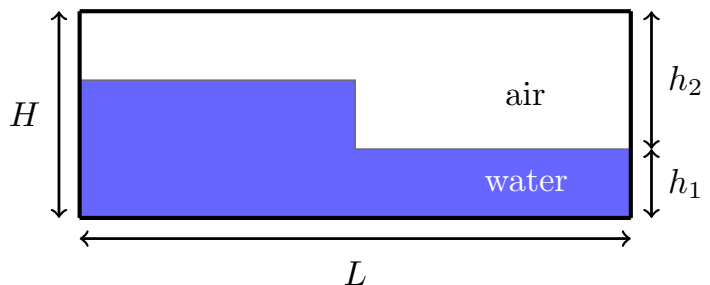

\begin{tabular}{|c|c|c|}
\hline Variable & $0 \leq x \leq L / 2$ & $L / 2<x \leq L$ \\
\hline$h_{1} / H$ & 0.6 & 0.4 \\
\hline$\rho_{1}$ & $998.1115 \mathrm{~kg} \cdot \mathrm{m}^{-3}$ & $998.1115 \mathrm{~kg} \cdot \mathrm{m}^{-3}$ \\
\hline$u_{1}$ & $0 \mathrm{~m} . \mathrm{s}^{-1}$ & $0 \mathrm{~m} . \mathrm{s}^{-1}$ \\
\hline$\rho_{2}$ & $1.204 \mathrm{~kg} \cdot \mathrm{m}^{-3}$ & $1.204 \mathrm{~kg} \cdot \mathrm{m}^{-3}$ \\
\hline$u_{2}$ & $0 \mathrm{~m} . \mathrm{s}^{-1}$ & $0 \mathrm{~m} . \mathrm{s}^{-1}$ \\
\hline
\end{tabular}

FIGURE 3.5. Initial conditions for the dambreak problem.

Contrary to the previous test case, the full system with pressure and velocity relaxation source terms is now involved. Regarding the SPR scheme, the relaxation parameters are set according to definition (2.11) for the stratified regimes $\left(h_{1}<H\right): a_{1}=0$ whereas $a_{2}$ follows the Whitham condition. The time step is computed using (3.1) for a given material CFL number denoted $\mathrm{CFL}_{\mathrm{m}}$.

The results obtained with a classical explicit Rusanov scheme are added for comparison. The latter is applied on the non-split convective part of $(\mathcal{S})$ while the source terms are treated in a second homogeneous step as in [35]. The corresponding time step is computed with an acoustic CFL number defined in (3.3) and set to $\mathrm{CFL}_{\mathrm{a}}=0.5$. Concerning the $\mathrm{SPR}$ scheme, it is assessed setting $\mathrm{CFL}_{\mathrm{m}}=0.5$ and $\mathrm{CFL}_{\mathrm{m}}=0.01$ which corresponds respectively to $\mathrm{CFL}_{\mathrm{a}}^{\prime} \sim 100$ and $\mathrm{CFL}_{\mathrm{a}}^{\prime} \sim 2$ for the considered dambreak problem.

\subsubsection{Results and comments}
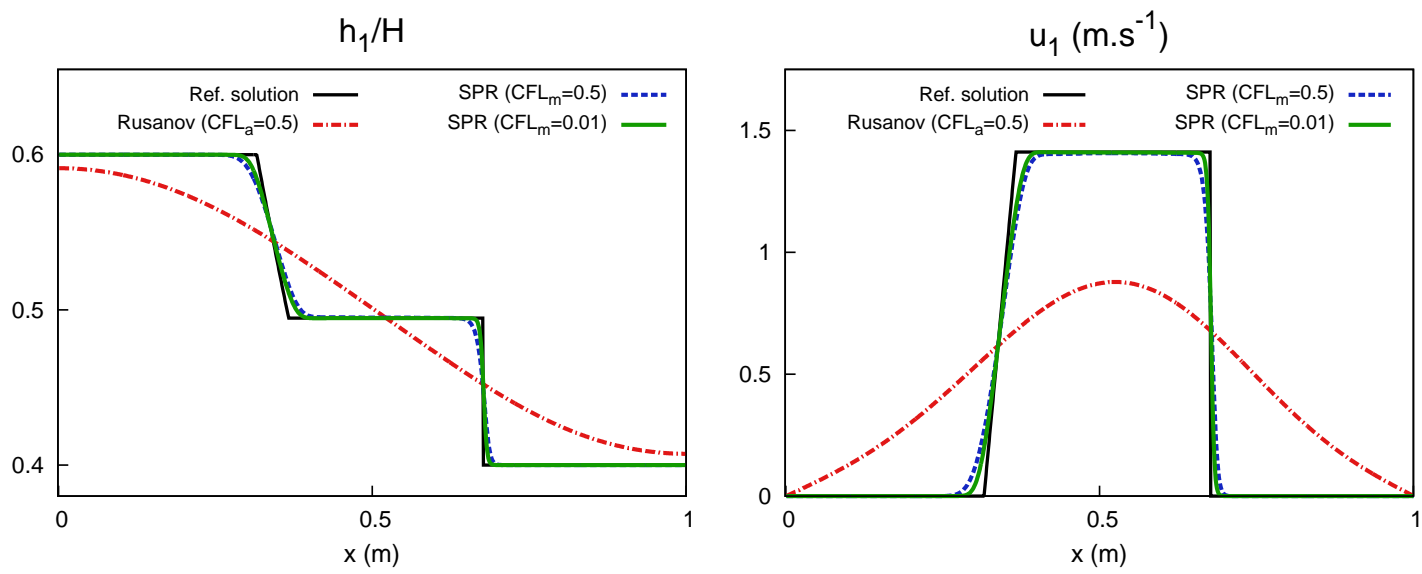

\begin{tabular}{|c|c|c|c|}
\hline Scheme & Rusanov with $\mathrm{CFL}_{\mathrm{a}}=0.5$ & SPR with $\mathrm{CFL}_{\mathrm{m}}=0.01$ & ${\text { SPR with } \mathrm{CFL}_{\mathrm{m}}=0.5}$ \\
\hline CPU time & $150 \mathrm{~s}$ & $30 \mathrm{~s}$ & $1 \mathrm{~s}$ \\
\hline
\end{tabular}

Figure 3.6. Approximate solutions and CPU times for the dambreak problem at $T=24 \cdot 10^{-2} \mathrm{~s}$ with 1000 cells.

The fields at $T=24 \cdot 10^{-2} s$ with 1000 cells and the corresponding CPU times are provided on Figure 3.6. The reference solution is accurately captured by the SPR scheme. It confirms the fact that the compressibility of water as well as the additional air layer have a minor influence here. The two CFL values yield close results 
but, in terms of efficiency, the best choice seems to be $\mathrm{CFL}_{\mathrm{m}}=0.5$. However, the expected behavior is obviously not captured by the Rusanov scheme which provides very diffusive results with large CPU time.

This test case illustrates the difficulties to simulate low speed stratified regimes, in the sense $\sqrt{g H} \ll c_{1}$, with the CTL model using a classical explicit scheme. The proposed SPR scheme is not only stable and convergent but also provides interesting results as it accurately captures the expected behavior with low CPU time. The next step is to handle mixed flow configurations which involve transitions between stratified and pressurized or dry regimes.

\subsection{Elementary mixed flow: a pipe filling}

An elementary test case involving transitions between stratified and pressurized regimes is considered. It is referred to as the pipe filling test case for which the presence of air has a minor influence.

\subsubsection{Global setting and objectives}

In the following, a sloping pipe is considered where the initial condition is a static condition with uniform water height, uniform density and zero speed, see Figure 3.7. A realistic rectangular pipe is chosen whose height $H$ is $0.2 \mathrm{~m}$, length is $2 \mathrm{~m}$, and $\theta$, its angle from the horizontal, is $-30^{\circ}$. In this framework, the CTL model is defined in the inclined frame and gravity source terms are added, see Appendix A.1 for details. The total simulation time is set to $T=2 s$ with wall boundary conditions (mirror states) at the inlet and outlet.

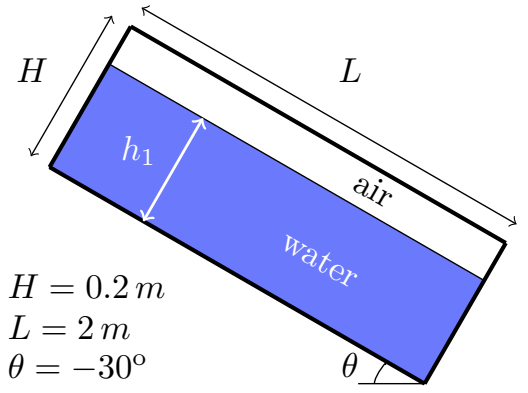

\begin{tabular}{|c|c|}
\hline Variable & $0 \leq x \leq 2 \mathrm{~m}$ \\
\hline$h_{1} / H$ & 0.8 \\
\hline$\rho_{1}$ & $998.1115 \mathrm{~kg} \cdot \mathrm{m}^{-3}$ \\
\hline$u_{1}$ & $0 \mathrm{~m} . \mathrm{s}^{-1}$ \\
\hline$\rho_{2}$ & $1.204 \mathrm{~kg} \cdot \mathrm{m}^{-3}$ \\
\hline$u_{2}$ & $0 \mathrm{~m} . \mathrm{s}^{-1}$ \\
\hline
\end{tabular}

FIgURE 3.7. Initial conditions for the pipe filling test case.

As this test case includes regime transitions, the adaptive stabilization process exposed in Section 2.4.3 is assessed hereafter. To say it briefly, stabilization terms for the water phase are activated when $h_{1} \geq h_{s}$, where $h_{s}=(1-\delta) H$ and $\delta=10^{-3}$. The time step is computed using (3.1) for a given material CFL number denoted $\mathrm{CFL}_{\mathrm{m}}$. The latter is set to 0.01 to get accuracy with fair efficiency as observed in the previous test cases, see also Remarks 2.15 and 3.1. In order to check the stability and the convergence of the method, a mesh refinement is performed considering regular meshes from 160 cells to 10240 cells, which corresponds to a space step range from $\Delta x \sim 1 \mathrm{~cm}$ to $\Delta x \sim 0.02 \mathrm{~cm}$.

The results obtained with the SPR scheme associated with the CTL model are compared with those obtained with the Pressurized Free Surface (PFS) model developed in [11]. This other model is also dedicated to mixed flows in pipes but it only computes the water phase, neglecting the presence of air. It is a one-layer model which couples the shallow-water equations in the free-surface regime with the isentropic Euler set of equations in the pressurized regime. At the discrete level, a Roe-type scheme is proposed in [11] and a kinetic scheme has also been recently derived in [10]. Both schemes are explicit in time with a CFL condition depending on the celerity of fast acoustic waves arising in the pressurized regime. The comparison between the two models is relevant as the PFS model is validated against several experimental data. Furthermore, the presence of air should have minor influence in the proposed configuration. 


\subsubsection{Results and comments}

Results with the CTL model. The evolution of the water height is depicted on Figure 3.8 through some snapshots along the simulation. The pipe is actually filling with a pressurized water front propagating towards the top of the pipe. When the front stops, one obtains a dry area $\left(h_{1} \simeq 0\right)$ and a pressurized area $\left(h_{1} \simeq H\right)$ separated by an oscillating free surface in the absence of wall friction effects. The fields at $t=0.3 \mathrm{~s}$ are detailed on Figure 3.9 with a mesh sensitivity analysis.

$\mathrm{t}=0.0 \mathrm{~s}$.

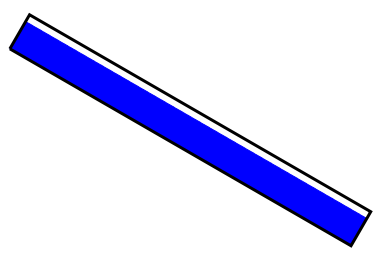

$\mathrm{t}=0.3 \mathrm{~s}$.

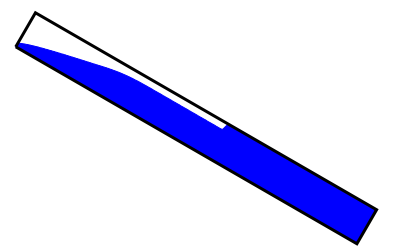

$\mathrm{t}=0.5 \mathrm{~s}$.

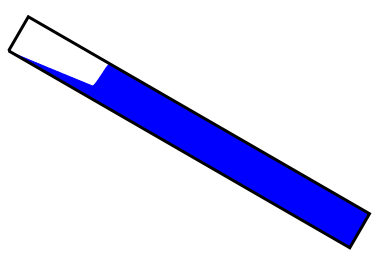

$\mathrm{t}=1.0 \mathrm{~s}$.

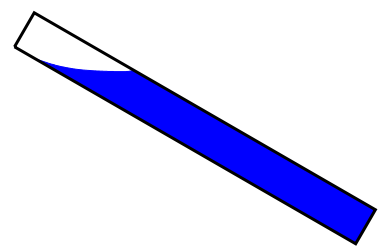

FIGURE 3.8. Snapshots of water height with 640 cells.
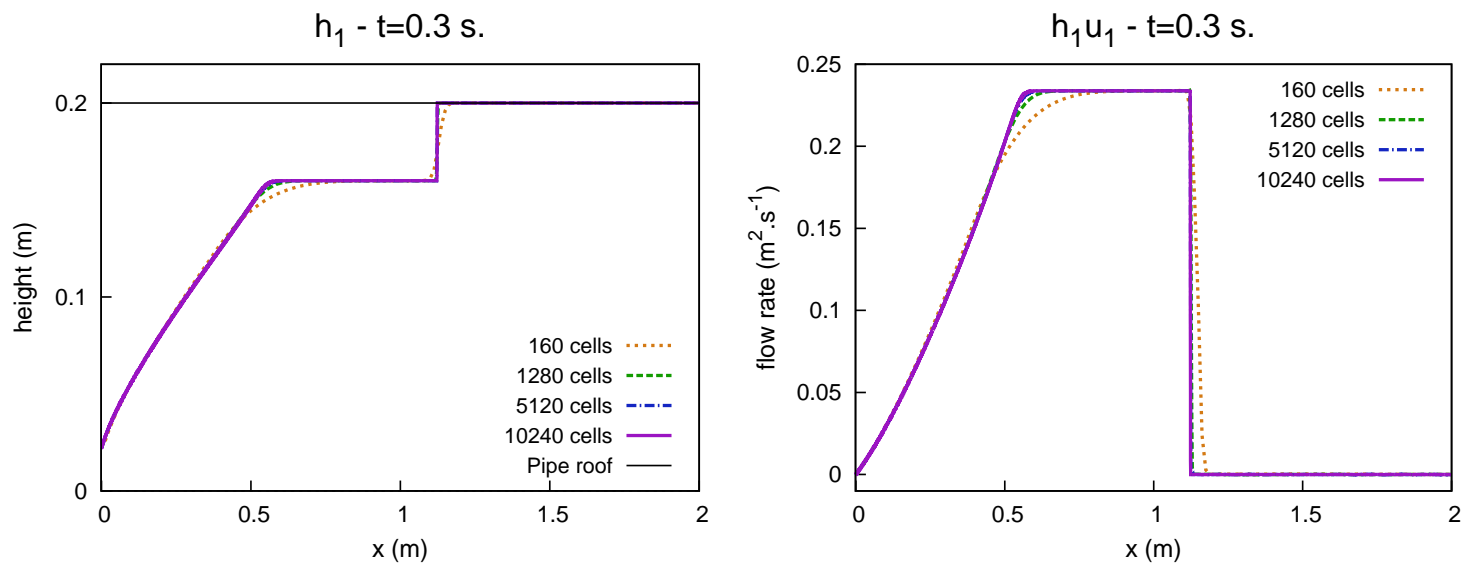

$h_{2} / H-t=0.3 s$.
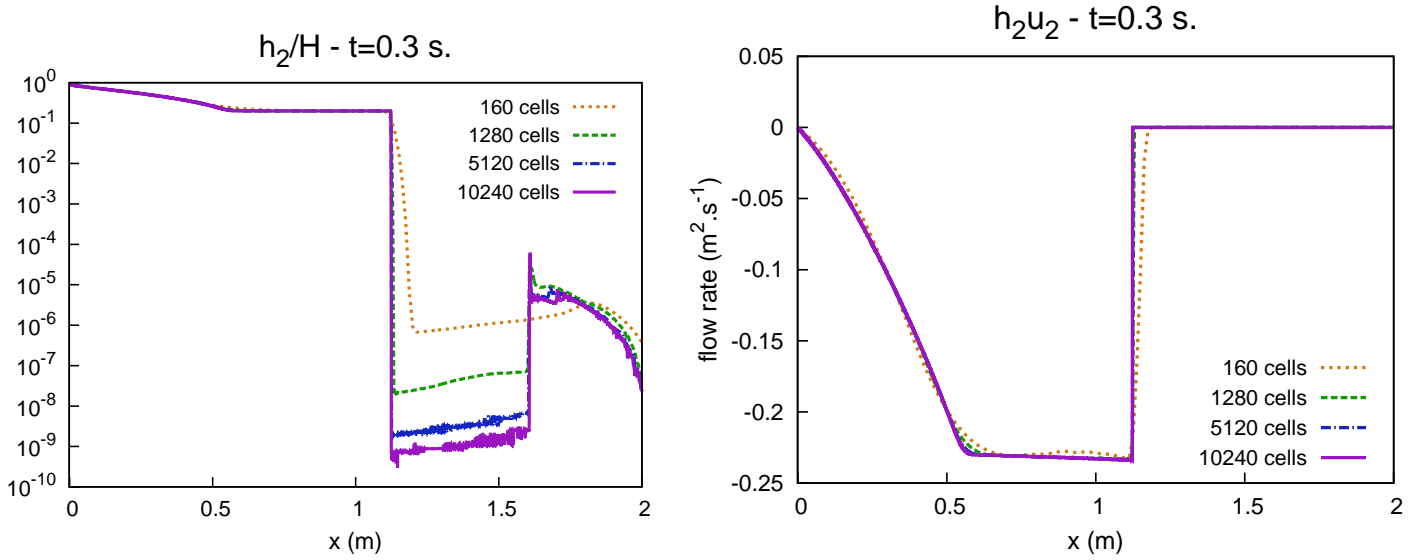

Figure 3.9. Approximate solutions for the pipe filling test case at $t=0.3 \mathrm{~s}$.

A mixed flow is obtained with a jump between the stratified and the pressurized part. The water height $h_{1}$, as well as the flow rate $h_{k} u_{k}, k=1,2$, display a fast mesh convergence. The air height field, depicted in 
$\log$ scale, illustrates the robustness of the SPR scheme regarding vanishing phases as $\frac{h_{2}}{H}$ may reach $10^{-9}$ in the pressurized part for the finest mesh. A very thin air layer is then solved but dissipative effects provided by the relaxation source terms bring stability. Note that the thickness of this air layer is independent of the threshold value $h_{s}$ as it tends to zero when the mesh is refined while the threshold is kept constant.

Focusing on the water pressure behavior on Figure 3.10, the mesh convergence appears more difficult to reach. On the left graph, i.e. the pressure field at $t=0.3 \mathrm{~s}$, spurious oscillations are observed at the transition point where the pressure jumps as well as in the pressurized part. Although these oscillations vanish when the mesh is refined, the corresponding numerical behavior is associated to the brutal transition in terms of wave speed between the stratified and the pressurized part, expressed by the ratio $\frac{\sqrt{g H}}{c_{1}} \sim 10^{-4} \ll 1$ in the present case. It is a common feature when dealing numerically with mixed flow, see [51] for a related study. Nonetheless, one obtains the expected slope in charge given by the equilibrium between the pressure gradient and gravity terms: $\partial_{x} P_{1}=-\rho_{1} g \sin (\theta)$. In addition, one may estimate the pressure jump value from Rankine-Hugoniot conditions, see Appendix B for details, which yield $\Delta P_{1} \sim 0.080$ bar while one measures $\Delta P_{1} \sim 0.081$ bar on the finest mesh. On the right graph of Figure 3.10, the water pressure time series at $x=1 \mathrm{~m}$ is plotted. Oscillations are also observed in time and they are attenuated with the mesh refinement. This time signal in charge is clarified hereafter using a lower acoustic wave celerity in the water phase.
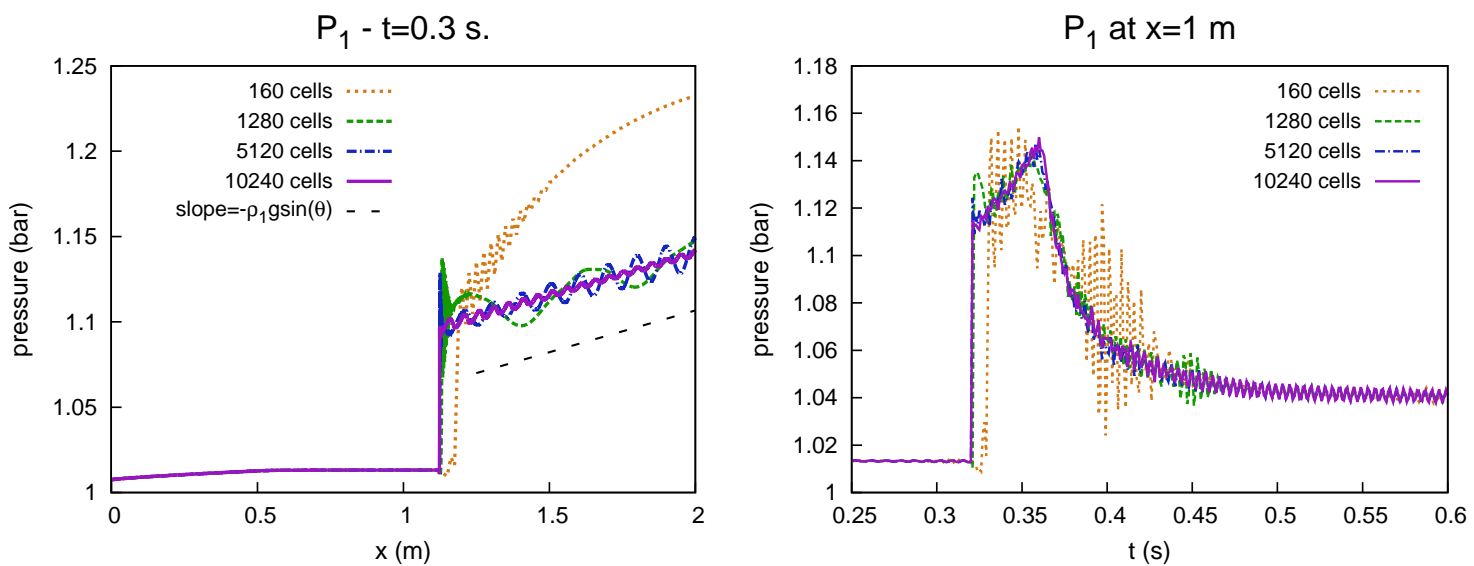

Figure 3.10. Water pressure field at $t=0.3 \mathrm{~s}$ (left) and time series at $x=1 \mathrm{~m}$ (right).

Influence of the acoustic waves celerity in the water phase. The celerity of acoustic waves in the water phase is given by $c_{1, \text { ref }}$ involved in the pressure law (1.2a). The reference value taken so far is $c_{1, \text { ref }}=1500 \mathrm{~m}_{\mathrm{s}} \mathrm{s}^{-1}$, corresponding to a pure water phase in a rigid pipe. In practice, dealing with air-water flows, the mixing between phases in addition to pipe elasticity effects may strongly reduce this celerity. Furthermore, a lower celerity may also smoothen the transition between the stratified and the pressurized regime. This classical and widely used trick is assessed hereafter setting $c_{1, \text { ref }}=200 \mathrm{~m}_{\mathrm{s}} \mathrm{s}^{-1}$, which matches with the order of magnitude experimentally measured in [15] for a PVC pipe of diameter $0.16 \mathrm{~m}$. The water pressure at $t=0.3 \mathrm{~s}$ as well as its time evolution at $x=1 m$ are presented on Figure 3.11 .

It is observed that the mesh convergence of the pressure field at $t=0.3 \mathrm{~s}$ is quite fast, canceling the spurious oscillations when the mesh is refined. The expected slope in charge is again well approximated. Regarding the pressure time series, the signal is also clarified. In particular, well structured oscillations are observed for $t \gtrsim 0.4 \mathrm{~s}$. Their period may be estimated considering the propagation of acoustic waves in the water medium. In the present case, it classically yields $T=\frac{4 L_{w}}{c_{1}}$ where $L_{w}$ denotes the pipe length filled by water, see [25] for instance. One analytically obtains $T=0.032 \mathrm{~s}$ while numerical approximations yield $T \sim 0.03 \mathrm{~s}$ on Figure 3.11. Regarding the amplitude, it seems to result from a combination between hydrostatic effects due to free surface oscillations and acoustic effects due to acoustic waves propagation. 

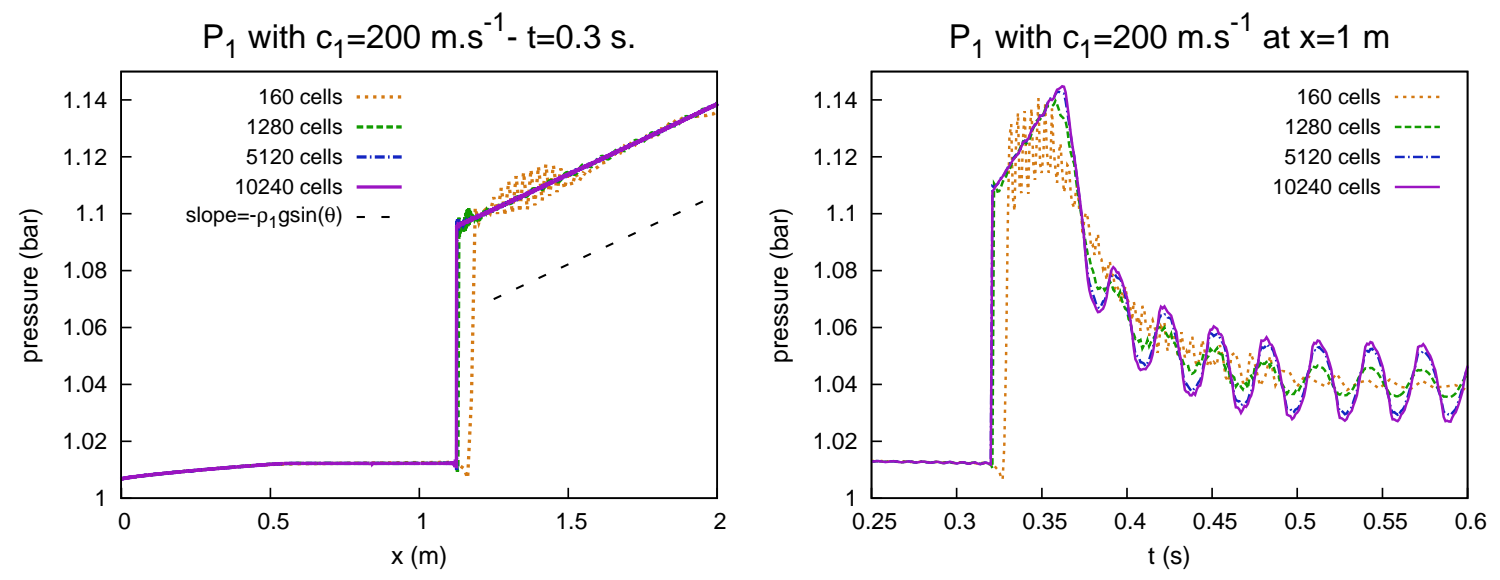

Figure 3.11. Water pressure field at $t=0.3 \mathrm{~s}$ (left) and time series at $x=1 \mathrm{~m}$ (right) using $c_{1}=200 \mathrm{~m} \cdot \mathrm{s}^{-1}$.

Note that when using $c_{1, \text { ref }}=1500 \mathrm{~m} \cdot \mathrm{s}^{-1}$, the correct period is also captured but with a weaker amplitude so that is does not clearly appear on Figure 3.10. The other fields, which concern heights and velocities, are not affected by this celerity change, nor the pressure jump in the present case. Finally, physically relevant and converged results are obtained using $c_{1, \text { ref }}=200 \mathrm{~m}_{\mathrm{s}} \mathrm{s}^{-1}$. They are compared hereafter with those obtained with the PFS model.

Comparison with the PFS model. As revealed on Figure 3.12, the CTL and the PFS models yield very close results on the same mesh (5120 cells) using $c_{1, \text { ref }}=200 \mathrm{~m} \cdot \mathrm{s}^{-1}$. This confirms that the air phase has a minor influence here. Furthermore, it also emphasizes the fact that the thin air layer computed with the CTL model in the pressurized part does not affect the pressurized dynamics. Regarding the pressure fields, the PFS model yields spurious oscillations at the transition point while the CTL model does not. It seems that the unified description of the CTL model in addition to its dissipative relaxation source terms brings more stability. Thus, in some sense, the additional air layer is beneficial.

Regarding the CPU times, it needs 20 minutes to compute the solution over 2 seconds on a 640 cells mesh with the CTL model. This computation time is independent of the celerity $c_{1}$ as the latter is not involved in the CFL condition (2.5). Using the explicit scheme developed in [10] for the PFS model, the CFL condition actually depends on $c_{1}$. A reduction of $c_{1}$ is therefore profitable for the PFS model in terms of CPU time. Thus, it requires 13 minutes when $c_{1}=1500 \mathrm{~m} . \mathrm{s}^{-1}$ and 2 minutes when $c_{1}=200 \mathrm{~m} . \mathrm{s}^{-1}$. The CTL model is naturally more computationally demanding as it involves a larger system with implicit parts. However, the CPU time is not prohibitive compared to the PFS model statistics. Furthermore, higher acoustic waves celerity can be chosen with more stability.

Lastly, the ability of the CTL model to handle mixed flows is highlighted with this test case. Indeed, one obtains physically relevant results in agreement with the PFS results and analytical results provided by a simplified approach. The CPU time is comparable with the one of the PFS and seems totally tractable for practical applications of industrial interest. Transitions between the regimes are correctly handled with the SPR scheme where dissipative effects ensured by relaxation source terms bring stability. In the next section, a more severe mixed flow test case is considered and the results are compared against experimental data. 

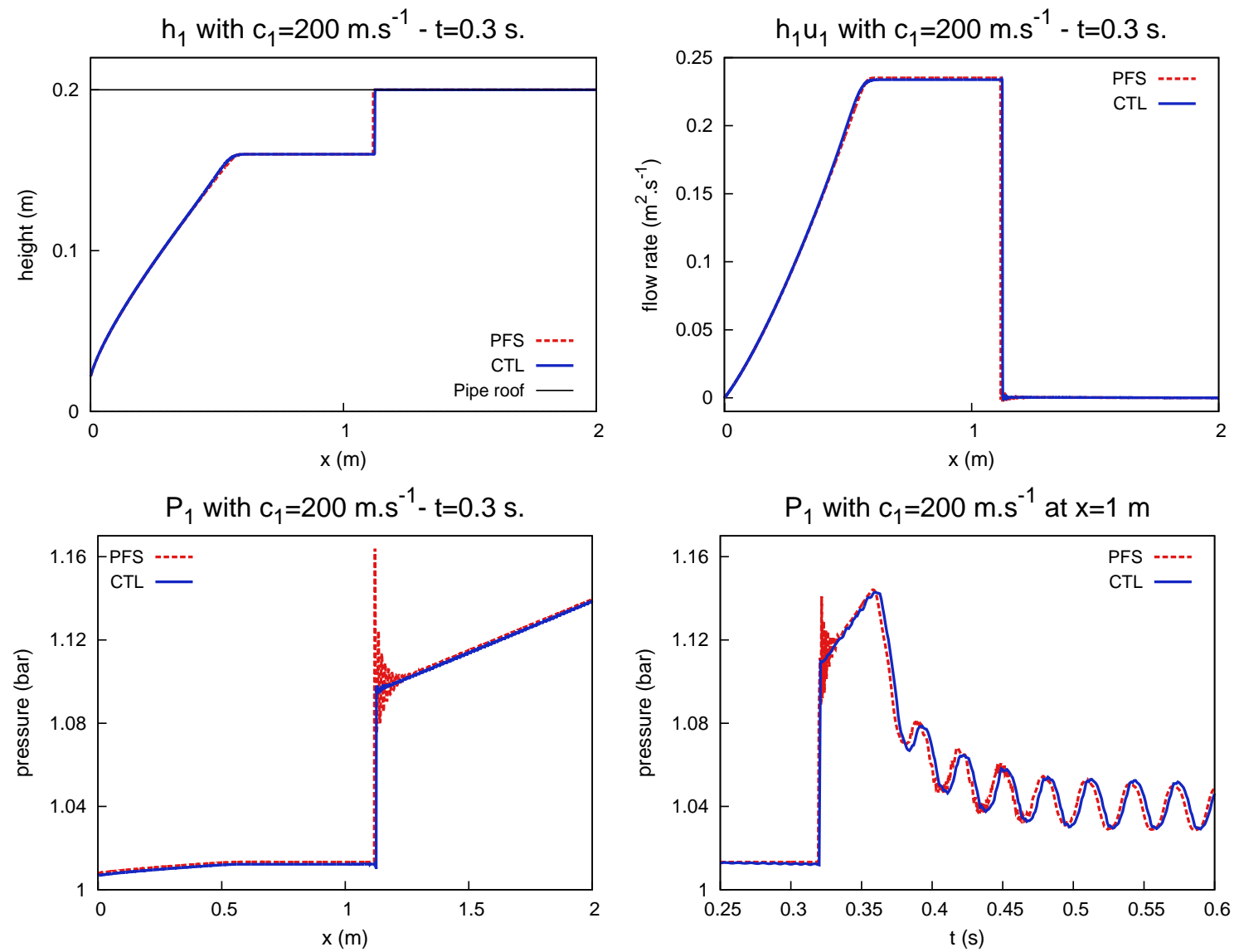

FIGURE 3.12. Comparison with the PFS model using $c_{1}=200 \mathrm{~m} . \mathrm{s}^{-1}$ and 5120 cells.

\subsection{Mixed flow with experimental validation: a laboratory test case (Aureli et al. (2015))}

In this section, a recent experimental test case dedicated to the validation of single-phase mixed flow models is considered, see [5]. The latter is closer to industrial applications as it involves a circular pipe with slope changes and several regime transitions. It can effectively be seen as a single-phase mixed flow due to the presence of vents at the upstream and downstream end of the pipe to avoid air pocket entrapment.

\subsubsection{Global setting and objectives}

The experimental configuration is depicted on Figure 3.13. It consists in a sloped PVC circular pipe of diameter $H=0.192 \mathrm{~m}$ and length $L=12.12 \mathrm{~m}$. A slope change occurs at $L_{1}=7 \mathrm{~m}$, switching from $\theta_{1}=-4.8^{\circ}$ to $\theta_{2}=15.48^{\circ}$. The pipe is opened at the inlet and outlet to avoid air pocket entrapment effects. Thus, atmospheric pressure is maintained at the two ends. The pipe is initially partially filled by water until a closed gate located at $x=5 \mathrm{~m}$ ( $x$ is the distance along the pipe axis). The experiment begins when the gate opens. It gives rise to a transient mixed flow with several transitions in both parts of the pipe. Note also that it involves dry areas. Pressure and velocity measurements are performed along the pipe at several locations during 30 seconds.

Dealing with circular pipes, the CTL model is slightly modified due to the $2 \mathrm{D}$ integration on a crosssection, see Appendix A.2 for details. Furthermore, friction effects occur between the pipe walls and the water phase. They are modeled by a Manning-Strickler law, which reads $F_{1}=-m_{1} g S_{f}$ where $S_{f}=n_{m}^{2} u_{1}\left|u_{1}\right| R_{h}^{-4 / 3}$, 


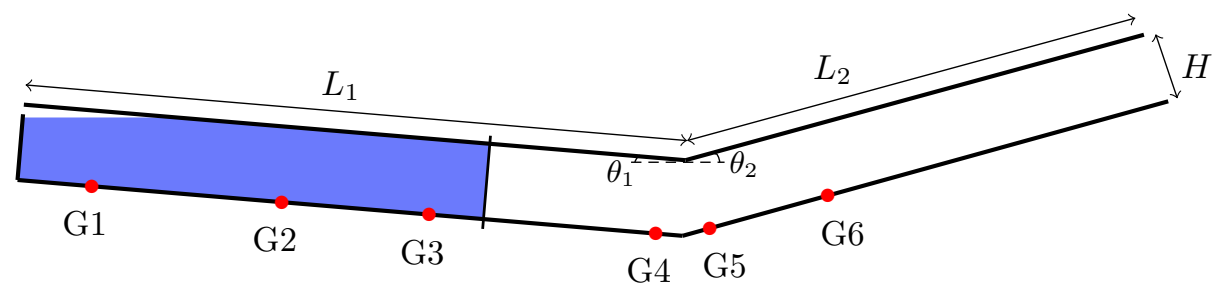

$$
\begin{aligned}
& L_{1}=7 m \\
& L_{2}=5.12 m \\
& H=0.192 m \\
& \theta_{1}=-4.8^{\circ} \\
& \theta_{2}=15.48^{\circ}
\end{aligned}
$$

Gk: pressure gauge location

Figure 3.13. Configuration and initial condition and for the Aureli et al. test case.

see [46]. In particular, the Manning roughness coefficient $n_{m}$ is set to $5.0 m^{-\frac{1}{3}} s$ and $R_{h}\left(A_{1}\right)=\frac{A_{1}}{P_{m}}$ is the so-called hydraulic radius where $P_{m}$ denotes the wet perimeter. The term $F_{1}$ is a source term of the momentum conservation equation for the water phase and is treated explicitly in a homogeneous additional step which occurs after the third step of the SPR scheme (velocity relaxation). The celerity of acoustic waves in the water phase is initially set to $c_{1, \text { ref }}=300 \mathrm{~m} . \mathrm{s}^{-1}$ in the linear pressure law $(1.2 \mathrm{a})$. The latter is physically relevant for the considered PVC pipe and makes the numerical convergence faster, see Section 3.3.

Regarding the boundary conditions, the air phase should be in equilibrium with the atmospheric pressure at the two ends. In practice, a vertical event is added at the left end of the pipe and periodic boundary conditions are imposed. This vertical event is only filled by air and acts as a wall for the water phase. The air phase is then maintained in equilibrium at both ends of the pipe and its initial value is given by the atmospheric pressure. For the other initial conditions, the water height is set to $h_{1}=\left(1-10^{-4}\right) H$ in the pressurized part

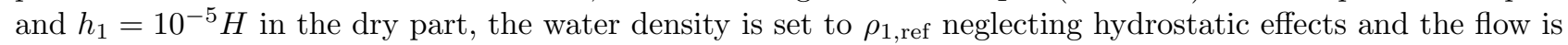
static. Focusing on the SPR scheme, the same setting as for the pipe filling test case of Section 3.3 is used. In particular, the threshold is set to $h_{s}=(1-\delta) H$ with $\delta=10^{-3}$ and the (material) CFL number is set to 0.01 . The same mesh size as in [5] is used, that is 300 cells which yields $\Delta x \sim 4 \mathrm{~cm}$.

\subsubsection{Results and comments}

The pressure head and velocity time series are displayed on Figure 3.14 for two relevant locations along the pipe. The following comments apply to the other locations whose results are provided in [25]. In the related paper [5], the pressure head is derived from pressure measurements assuming an hydrostatic pressure distribution over the pipe section. In the CTL framework, the latter is denoted $\mathcal{H}$ and defined as:

$$
\mathcal{H}=h_{1}+\frac{P_{1}-P_{\text {hydro }}}{\rho_{1, \text { ref }} g}
$$

where $P_{\text {hydro }}$ corresponds to the supposed mean hydrostatic pressure on a cross section. It writes $P_{h y d r o}=$ $P_{a t m}+\rho_{1, \text { ref }} g \ell_{1} \cos (\theta)$ for circular pipes where $P_{a t m}$ is the air pressure at the outlet (or inlet) and $\ell_{1}$ is the distance between the free surface and the center of mass of the wet section, see [27]. In the stratified regime, $\rho_{1} \sim \rho_{1, \text { ref }}$ and $P_{2} \sim P_{\text {atm }}$, such that $P_{1}-P_{\text {hydro }}$ may be rewritten as $P_{I}-P_{2} \sim 0$ due to pressure relaxation. Therefore, $\mathcal{H} \sim h_{1}$ in the stratified regime. In the pressurized regime, $\mathcal{H}$ computes the equivalent height corresponding to an overpressure or subpressure in comparison to the hydrostatic reference.

As displayed on Figure 3.14, the CTL model is able to reproduce faithfully the experimental observations on pressure head and velocity time series. Indeed, the pressure heads in both stratified and pressurized regimes are correctly captured. Transitions between the regimes are also particularly well apprehended. Even if the authors of [5] temper about the quality of velocity measurements, the obtained numerical results also highlight the good behavior of the CTL model. Furthermore, the required CPU time to perform a 30 seconds simulation on a single processor is 25 minutes, which seems reasonable regarding the accuracy of the results and the complexity of the model.

Comparable numerical results are obtained in [5] when using single-phase mixed flow models, i.e. the PFS model [11] and the Preissmann slot model [24]. However, the authors illustrate the lack of robustness of the 
latter models when using a physically relevant acoustic wave celerity. Indeed, the latter is set to $c_{1, \text { ref }}=12 m . s^{-1}$ in [5] to get satisfactory results whereas $c_{1 \text {,ref }}=200 \mathrm{~m} . \mathrm{s}^{-1}$ leads to strong spurious oscillations, in particular with the Preissmann slot model. This drawback is tempered in the related paper as only a weak influence of this celerity value is identified. Nonetheless, when dealing with longer pipes encountered in industrial configurations, typically $L \sim 100 \mathrm{~m}$, such a low value of $c_{1, \text { ref }}$ may provide non physical results.
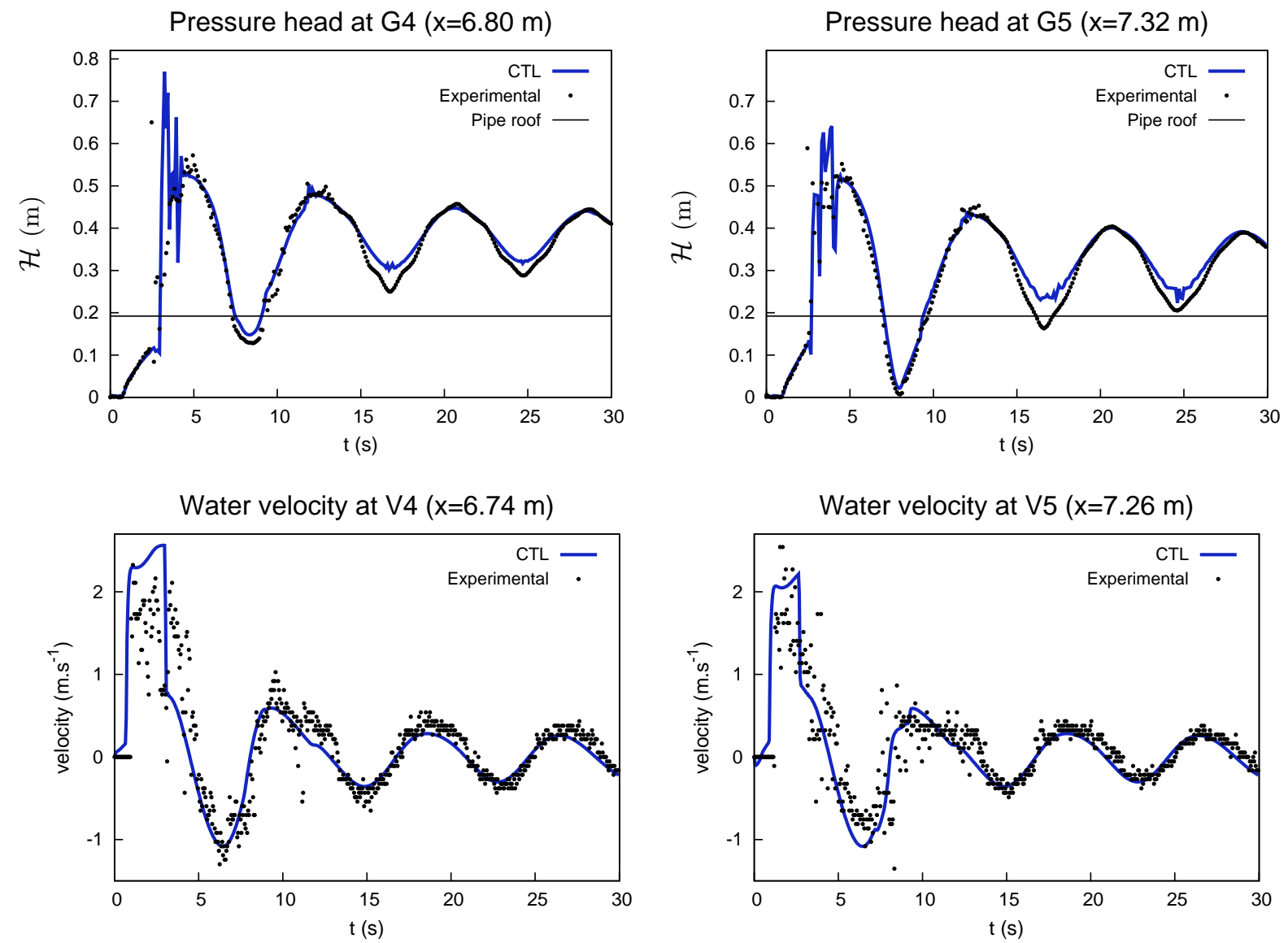

Figure 3.14. Comparison between experimental and numerical results using 300 cells.

The results obtained for this experimental test case validate the CTL model in the configuration of mixed flow where the air phase has a weak influence. Indeed, pressure heads and velocity time series are correctly captured at several locations along the pipe where transitions from stratified to pressurized regimes and vice versa occur. Furthermore, the stability and the efficiency of the SPR scheme are also highlighted as a high acoustic wave celerity in the water phase may be chosen with non prohibitive CPU time. In the next section, a test case involving air pocket entrapment is considered.

\subsection{Mixed flow with air pocket entrapment: a U-Tube test case}

In this section, the ability of CTL model to handle air pocket entrapment and air pressurization is assessed. A two-phase mixed flow configuration is thus considered through a closed pipe describing a U-shape filled by water and air. In particular, the air phase is pressurized and entrapped at the two ends of the pipe. The case where the pipe is open at the boundaries is also considered. 


\subsubsection{Global setting and objectives}

The configuration under consideration is the U-Tube pipe depicted on Figure 3.15. Realistic dimensions are chosen with $H=0.1 \mathrm{~m}, L=4.5 \mathrm{~m}$ and $D=1 \mathrm{~m}$. The water elevation is measured from the two ends of the pipes, respectively $a$ from the left side and $b$ for the right side. The air pressure is denoted $P_{2}^{l}(t)$ in the left air pocket and $P_{2}^{r}(t)$ in the right one. In the initial state, the flow is kept static. This system may enter in an oscillatory mixed flow regime depending on initial air pressures $\left(P_{2,0}^{l}, P_{2,0}^{r}\right)$ and water elevations $\left(a_{0}, b_{0}\right)$. It is proposed to handle this configuration with the CTL model studying the influence of the initial state. A reference solution is derived in Appendix C.

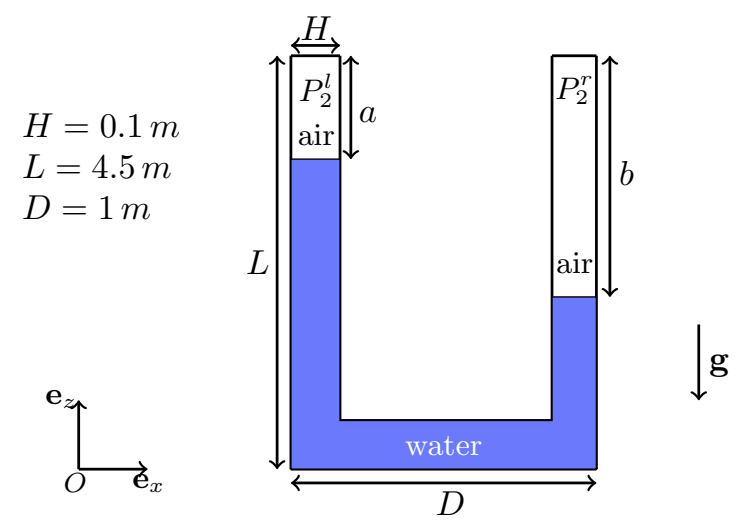

Figure 3.15. Geometric description of the U-Tube test case.

Therefore, three different settings detailed on Table 1 are considered. Initial water elevations are identical while initial air pressures and boundary conditions vary. Air pockets with different pressures are thus entrapped in the $\mathrm{C} 1$ and $\mathrm{C} 2$ settings while the $\mathrm{OP}$ setting refers to an open pipe without any air pocket entrapment. For the other initial conditions, the water height is set to $h_{1}=\left(1-10^{-4}\right) H$ in the pressurized part and $h_{1}=10^{-5} H$

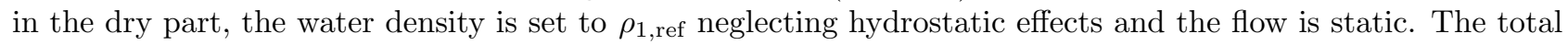
simulation time is set to 10 seconds.

\begin{tabular}{|c|c|c|c|c|c|}
\hline Id & $P_{2,0}^{l}$ & $P_{2,0}^{r}$ & $a_{0}$ & $b_{0}$ & BCs \\
\hline C1 & $1.1 \mathrm{~atm}$ & $1 \mathrm{~atm}$ & $0.3 L$ & $0.6 L$ & Wall \\
\hline $\mathrm{C} 2$ & $1.8 \mathrm{~atm}$ & $1 \mathrm{~atm}$ & $0.3 L$ & $0.6 L$ & Wall \\
\hline OP & $1 \mathrm{~atm}$ & $1 \mathrm{~atm}$ & $0.3 L$ & $0.6 \mathrm{~L}$ & Periodic \\
\hline
\end{tabular}

TABLE 1. Initial and boundary conditions for U-Tube test cases.

In practice, the CTL model is defined along the pipe axis, see Appendix A.1, without any treatment for the slope change, simply accounted for by a discontinuity on $\theta$. Note that the CTL model is well defined for $\theta \in\left[-\frac{\pi}{2}, \frac{\pi}{2}\right]$ such that right angle bends may be handled. The celerity of acoustic waves in the water phase is set to $c_{1, \text { ref }}=1500 m^{-1}$. Focusing on the SPR scheme, the same setting as for the previous mixed flow test cases is used. In particular, the threshold is set to $h_{s}=(1-\delta) H$ with $\delta=10^{-3}$ and the (material) CFL number is set to 0.01. A regular mesh composed of 300 cells $(\Delta x \sim 3.3 \mathrm{~cm})$ is chosen and the numerical results are compared with the reference solution derived in Appendix $\mathrm{C}$. 


\subsubsection{Results and comments}

For each setting exposed on Table 1, the air pressure at $x=0$ (i.e. $P_{2, l}$ ) as well as the water velocity at $x=L_{t} / 2$ (middle of the pipe) are evaluated with the CTL model. The results are displayed on Figure 3.16. The CTL model compares well with the reference solution both in terms of amplitude and frequency for all settings. Due to numerical diffusion, a slight shift associated to an amplitude damping is observed in the long time evolution, particularly for the most severe setting corresponding to C2. This numerical diffusion naturally diminishes when using a finer mesh, see [25] for details.
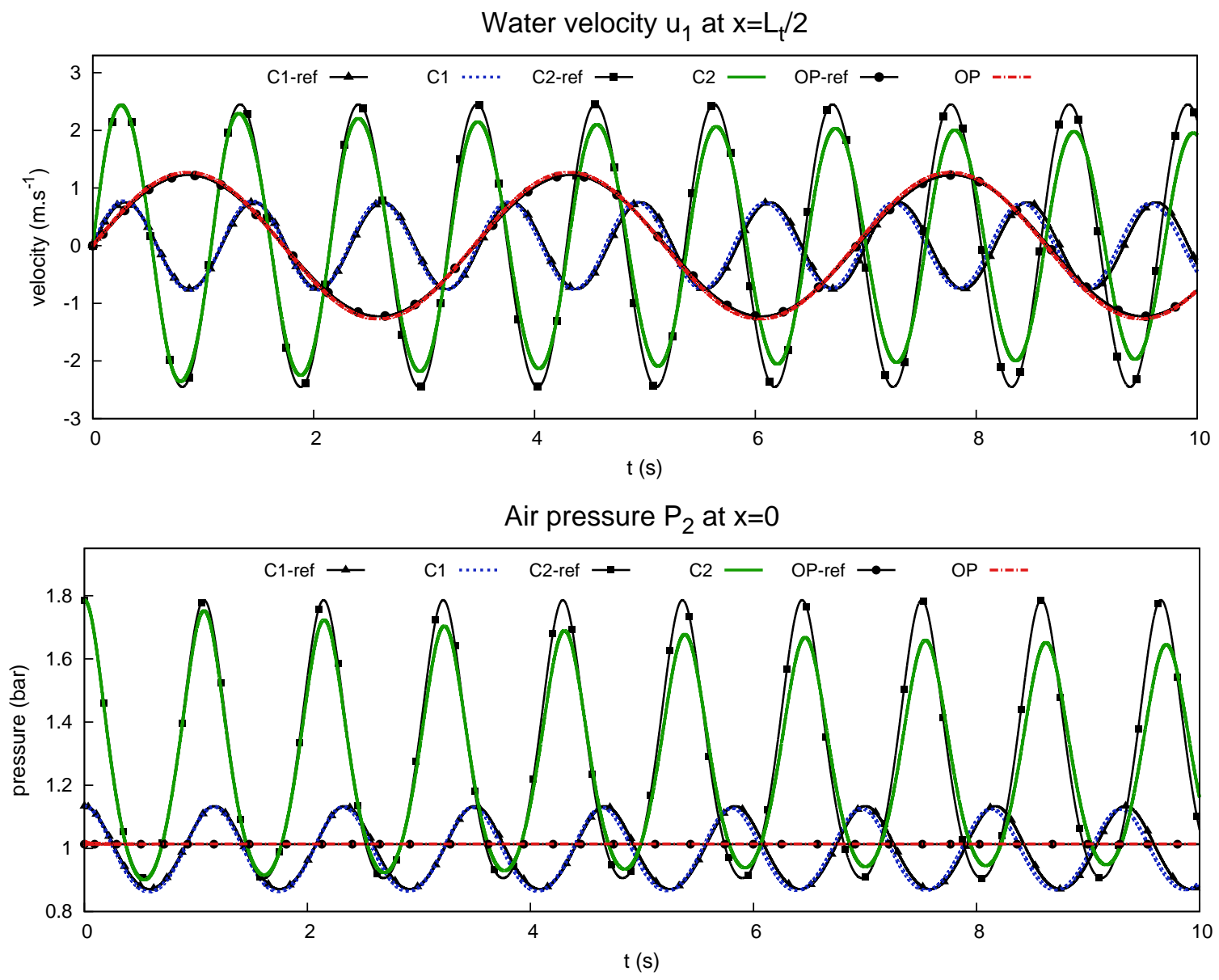

Figure 3.16. Comparison between the reference solution and numerical results for all settings using 300 cells.

These results illustrate the ability of the CTL model to handle air pocket entrapment in pipes with different degrees of pressurization. This last feature is a key point regarding the two-phase modelling of mixed flows. Indeed, as highlighted on Figure 3.16, the solution strongly depends on the air pressurization level where a large disparity both in terms of frequency and amplitude is observed.

\section{Conclusion}

The numerical resolution of the Compressible Two-Layer model is addressed in this paper with the aim of simulating mixed flows and entrapped air pockets in pipes. The main properties of this model are first recalled 
highlighting its relevance for such flows. In particular, the CTL model is an hyperbolic five-equation model which provides a unified two-phase description of the different regimes. At the discrete level, a robust splitting method combined with an implicit-explicit time discretization is proposed. The latter relies on the fast pressure relaxation in addition to a mimetic approach with the shallow water equations for the slow dynamics of the water phase. It results in a three-step scheme, namely the SPR scheme, where the slow dynamics of the system is treated explicitly whereas the fast dynamics is treated implicitly. The slow dynamics includes the water hydrostatic gradient which corresponds to a driving term in the stratified regime. The fast dynamics includes the pressure gradients in a second sub-system for which an original implicit relaxation-like method is proposed. It ensures the stable resolution of acoustic waves through additional diffusion terms. For the water phase, these terms are only activated in the pressurized regime according to a criterion on the water height. The third subsystem deals with the velocity relaxation using a classical implicit approach. For the three steps, a particular attention is paid to the robustness in the presence of vanishing phases ensuring discrete relaxation processes and non-singular systems to solve. Finally, the positivity of heights and densities is guaranteed under a CFL condition based on the celerity of material and gravity waves.

The stability and the convergence of the method towards relevant shock solutions is verified on a Riemann problem for the convective part of the model. Including the relaxation source terms, the proposed scheme accurately captures the expected behavior on a dambreak problem. Then, the three test cases presented clearly illustrate the ability of the Compressible Two-Layer model associated with the SPR scheme to deal with mixed flows in pipes including air-water interactions. The efficiency and the robustness of the method regarding transitions between the regimes are highlighted and it is important to note that these attractive features are obtained with a two-phase description in every regime, particularly in pressurized and dry regimes where a thin-layer of vanishing fluid is computed.

To the best knowledge of the authors, the CTL model is thus the only validated 1D two-phase model for mixed flows in pipes providing an accurate compressible description of both phases in every regime. Therefore, numerous two-phase transient mixed flow configurations including air pockets entrapment seem achievable. In that sense, the current work involves the development of boundary conditions which is still an open problem for two-phase two-pressure models in the general case due to the complexity of the waves structure.

This work has been partially funded by ANRT and EDF through an EDF-CIFRE contract 749/2014. Computational facilities were provided by EDF.

\section{Appendix A. Sloping PIPES}

\section{A.1. Sloping rectangular pipes}

Sloping pipes are frequently encountered in industrial configurations. Considering a constant slope of angle $\theta \in\left[-\frac{\pi}{2} ; \frac{\pi}{2}\right]$, a description of the geometry is presented on Figure A.1.

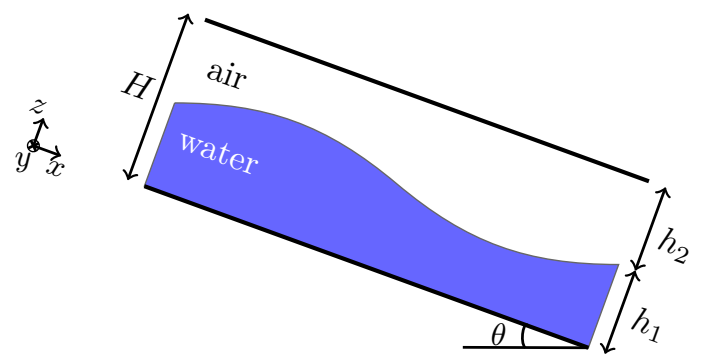

Figure A.1. Geometric description for sloping pipes. 
The frame of reference is the inclined frame $(O, x, y, z)$ so that the closure relations (1.1) for interfacial variables readily become:

$$
\left(U_{I}, P_{I}\right)=\left(u_{2}, P_{1}-\rho_{1} g \frac{h_{1}}{2} \cos (\theta)\right)
$$

Thus, gravity source terms are also added in the momentum conservation equations of the CTL model which now write:

$$
\partial_{t} m_{k} u_{k}+\partial_{x} m_{k} u_{k}^{2}+\partial_{x} h_{k} P_{k}\left(\rho_{k}\right)-P_{I} \partial_{x} h_{k}=(-1)^{k} \lambda_{u}\left(u_{1}-u_{2}\right)-m_{k} g \sin (\theta), \quad k=1,2
$$

At the discrete level, the gravity source terms are treated implicitly in the first step of the SPR scheme, see Section 2.3. The partial masses $m_{k}^{*}$ are indeed available from the mass conservation equations. If the slope of the pipe is varying in space, i.e. $\theta(x)$, one still uses (A.2) imposing a discontinuity on $\theta$ at the discrete level without any additional treatment. In particular, curvature effects are not taken into account.

\section{A.2. Sloping circular pipes}

In the framework of circular pipes, the CTL model results from a 2D integration of the isentropic Euler set of equations for both phases over a cross-section. The momentum conservation equations read as (A.2) except that $h_{k}$ is replaced by $A_{k}$, the area filled by the phase $k$ in the cross section and the interfacial closure laws becomes:

$$
\left(U_{I}, P_{I}\right)=\left(u_{2}, P_{1}-\rho_{1} g \ell_{1}\right) .
$$

The length $\ell_{1}$ represents the distance between the interface and the center of mass of the wet section, it writes for circular pipes:

$$
\ell_{1}=\frac{R^{3}}{A_{1}}\left(\frac{2}{3} \sin ^{3} \frac{\theta_{1}}{2}-\frac{1}{2} \cos \frac{\theta_{1}}{2}\left(\theta_{1}-\sin \theta_{1}\right)\right),
$$

where $A_{1}=\frac{R^{2}}{2}\left(\theta_{1}-\sin \left(\theta_{1}\right)\right)$ and $\mathrm{R}$ is the radius of the pipe, see [27] for details.

\section{Appendix B. Estimation of the PREssure Jump FOR THE PIPE Filling test CASE}

The pipe filling test case addressed in Section 3.3 is characterized by a jump between the stratified and the pressurized part. In particular, on may estimate the related pressure jump for the water phase. The state close to the jump location in the stratified part is denoted $\mathbf{W}^{-}$while the state in the pressurized part is denoted $\mathbf{W}^{+}$. Denoting $\sigma$ the speed of the jump, the Rankine-Hugoniot jump conditions applied to the mass conservation equation in the water phase, see $(\mathcal{S})$, yield:

$$
\sigma=\frac{\left[\rho_{1} h_{1} u_{1}\right]_{-}^{+}}{\left[\rho_{1} h_{1}\right]_{-}^{+}}
$$

where the brackets [.] denote the difference between the states on both sides of the discontinuity, see [34]. It is

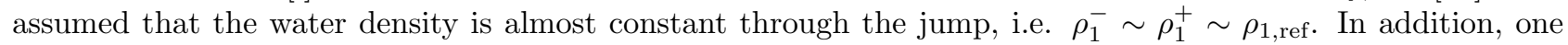
has $u_{1}^{+}=0$ and $h_{1}^{+}=H$ such that:

$$
\sigma=\frac{h_{1}^{-}}{h_{1}^{-}-H} u_{1}^{-} .
$$

The same approach on the air phase where $h_{2}^{+}=0$ and $u_{2}^{+}=0$ yields:

$$
\sigma=u_{2}^{-}
$$

Summing the momentum conservation equation of each phase, a conservative equation is obtained where the Rankine-Hugoniot jump conditions yield:

$$
-\sigma\left[m_{1} u_{1}+m_{2} u_{2}\right]_{-}^{+}+\left[m_{1} u_{1}^{2}+m_{2} u_{2}^{2}+h_{1} P_{1}+h_{2} P_{2}\right]_{-}^{+}=0 .
$$


In the pressurized part, one has $u_{1}^{+}=u_{2}^{+}=0, h_{2}^{+}=0$, such that we are left with:

$$
\left(m_{1} u_{1}\right)^{-}\left(\sigma-u_{1}^{-}\right)+\left(m_{2} u_{2}\right)^{-}\left(\sigma-u_{2}^{-}\right)+H P_{1}^{+}-\left(\left(h_{1} P_{1}\right)^{-}+\left(h_{2} P_{2}\right)^{-}\right)=0 .
$$

An instantaneous pressure relaxation is assumed in the stratified part such that one obtains $P_{2}^{-}=P_{I}^{-}=$ $P_{1}^{-}-m_{1}^{-} \frac{g}{2} \cos (\theta)$. Denoting $\Delta P_{1}=P_{1}^{+}-P_{1}^{-}$and using (B.2) and (B.3), (B.5) provides:

$$
\Delta P_{1}=\rho_{1}^{-}\left(\frac{h_{1}^{-}}{H-h_{1}^{-}}\left(u_{1}^{-}\right)^{2}-\frac{g}{2} \frac{h_{1}^{-}\left(H-h_{1}^{-}\right)}{H} \cos (\theta)\right)
$$

where one reasonably assumes $\rho_{1}^{-}=\rho_{1 \text {,ref }}$ and $h_{1}^{-}=h_{1, \text { init }}$. The value $u_{1}^{-}$may be estimated considering that the flow in the stratified part is uniform along $x$ close to the jump. Thus, neglecting the friction with the air phase, $u_{1}^{-}$complies with $\partial_{t} u_{1}^{-}=-\rho_{1} g \sin (\theta)$ which yields $u_{1}^{-}(t)=-\rho_{1} g t \sin (\theta)$. Therefore, (B.6) provides an analytical estimation of the pressure jump $\Delta P_{1}$. Note that this estimation does not involve the air phase nor the celerity of acoustic waves in the water phase.

\section{Appendix C. Reference solution for the U-Tube test CASe}

A reference solution for the so-called U-Tube test case is derived in this appendix. This derivation is proposed in a general framework where the sloped branches of the pipe are inclined from an angle $\theta$ compared to the horizontal reference, as depicted on Figure C.1 $\left(\theta=\frac{\pi}{2}\right.$ for the U-Tube). Thus, the configuration under consideration consists in a symmetric closed rectangular pipe of total length $L_{t}$ and height $H$ describing a V-shape filled by water and air. The length of both sloped parts of the pipe is denoted $L$ and the center part length is denoted $D$ such that $L_{t}=D+2 L$.

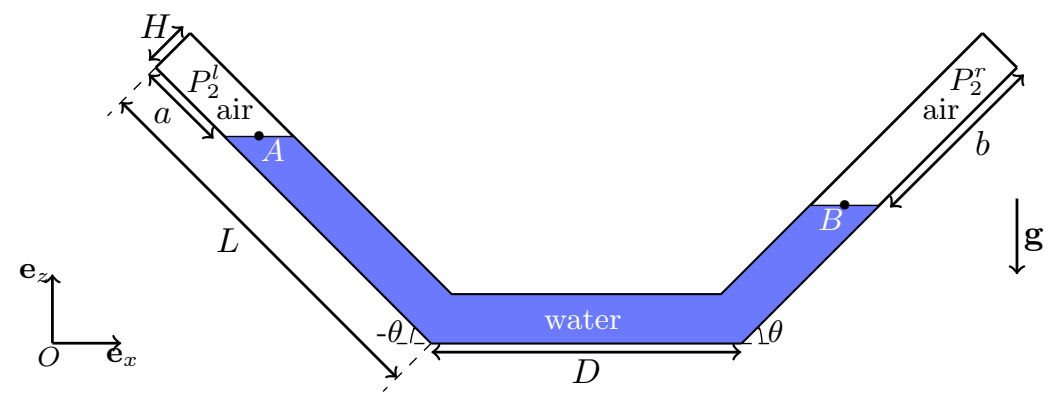

Figure C.1. Geometric description of the V-Tube.

The water elevation is measured from the two ends of the pipes, respectively $a$ from the left side and $b$ for the right side. The air pressure is denoted $P_{2}^{l}(t)$ in the left air pocket and $P_{2}^{r}(t)$ in the right one. In the initial state, the flow is kept static. This system may enter in an oscillatory mixed flow regime depending on initial air pressures $\left(P_{2,0}^{l}, P_{2,0}^{r}\right)$ and water elevations $\left(a_{0}, b_{0}\right)$.

The unsteady equation verified by $a(t)$ is derived hereafter. To this aim, a thin pipe compared to its length is considered, i.e. $H \ll L_{t}$, so that a $1 \mathrm{D}$ model is built assuming that the water phase is incompressible. It is also assumed that the water phase cannot touch the boundary walls, i.e. the water elevations remain positive: $a(t)>0, b(t)>0, \forall t$. In the reference frame $\left(0, \mathbf{e}_{x}, \mathbf{e}_{z}\right)$ and neglecting friction effects, the incompressible Euler 
set of equations writes:

$$
\begin{aligned}
& \operatorname{div}\left(\mathbf{v}_{\mathbf{1}}\right)=0 \\
& \frac{\partial \mathbf{v}_{1}}{\partial t}+\nabla\left(\frac{v_{1}^{2}}{2}\right)+\operatorname{rot} \mathbf{v}_{1} \wedge \mathbf{v}_{1}+\frac{1}{\rho_{1, \text { ref }}} \nabla P_{1}=\mathbf{g}
\end{aligned}
$$

where $\mathbf{v}_{1}$ is the local velocity vector of the water phase $\left(v_{1}^{2}=\mathbf{v}_{\mathbf{1}} \cdot \mathbf{v}_{\mathbf{1}}\right), P_{1}$ the local pressure, $\rho_{1, \text { ref }}$ the water density assumed constant and $\mathbf{g}$ the gravity field.

Let be $A$ and $B$, two points belonging to the free surface on each side of the pipe. The momentum conservation equation (C.1b) is integrated along a streamline going from A to B oriented by an elementary displacement vector $\mathbf{d l}=d x \mathbf{e}_{x}+d z \mathbf{e}_{z}$. By definition, $\mathbf{v}_{1} \wedge \mathbf{d} \mathbf{l}=\mathbf{0}$, such that it provides:

$$
\int_{A}^{B} \frac{\partial \mathbf{v}_{1}}{\partial t} \cdot \mathbf{d} \mathbf{l}+\int_{A}^{B} \nabla\left(\frac{v_{1}^{2}}{2}+\frac{P_{1}}{\rho_{1, \mathrm{ref}}}+g z\right) \cdot \mathbf{d} \mathbf{l}=0
$$

which can be rewritten as:

$$
\int_{A}^{B} \frac{\partial \mathbf{v}_{1}}{\partial t} \mathbf{d} \mathbf{l}+\frac{v_{1}^{2}\left(z=z_{B}^{-}\right)-v_{1}^{2}\left(z=z_{A}^{-}\right)}{2}+\frac{P_{1}\left(z=z_{B}^{-}\right)-P_{1}\left(z=z_{A}^{-}\right)}{\rho_{1, \text { ref }}}+g\left(z_{B}-z_{A}\right)=0 .
$$

The $z$ coordinates are given by $z_{A}=(L-a) \sin (\theta)$ and $z_{b}=(L-b) \sin (\theta)$ where $L$ is the length of both sloped part of the pipe. The water phase is considered in pressure equilibrium with the air phase at the free-surface such that $P_{1}\left(z=z_{A}^{-}\right)=P_{2}\left(z=z_{A}^{+}\right)$and $P_{1}\left(z=z_{B}^{-}\right)=P_{2}\left(z=z_{B}^{+}\right)$(surface tension effects are neglected here). Furthermore, the air pressure is assumed uniform in each air pocket. Due to the incompressible framework, the divergence free condition (C.1a) yields a uniform flow rate along the pipe. In particular, for a thin pipe with constant cross-section, it is assumed that:

$$
\mathbf{v}_{1}=\dot{a} \mathbf{u}
$$

everywhere in the water medium where $\mathbf{u}$ is a unitary vector oriented by $\mathbf{d l}$. Therefore, $v_{1}^{2}\left(z=z_{B}^{-}\right)=v_{1}^{2}\left(z=z_{A}^{-}\right)$ and (C.3) provides:

$$
L_{w} \ddot{a}+g(a-b) \sin (\theta)+\frac{P_{2}^{r}-P_{2}^{l}}{\rho_{1, \text { ref }}}=0
$$

where $L_{w}$ length is the length of a streamline connecting $A$ with $B$. For a thin pipe, it is assumed that $L_{w}=L_{t}-(a+b)$. The mass conservation of the water phase in the incompressible framework writes:

$$
a(t)+b(t)=a_{0}+b_{0}, \forall t
$$

Regarding the air phase, the air pockets are assumed to be kept separated by the water medium. Therefore, the mass conservation applies in each of them and yields $\rho_{2}^{l} a=\rho_{2,0}^{l} a_{0}$ and $\rho_{2}^{r} b=\rho_{2,0}^{b} b_{0}$ due to the uniform pressure assumption. Using a prefect gas law for the air phase, see (1.2b), one obtains:

$$
\left\{\begin{array}{l}
P_{2}^{l}=P_{2,0}^{l}\left(\frac{a_{0}}{a}\right)^{\gamma_{2}} \\
P_{2}^{r}=P_{2,0}^{r}\left(\frac{b_{0}}{b}\right)^{\gamma_{2}}
\end{array}\right.
$$

Denoting $l_{0}=a_{0}+b_{0}, a(t)$ finally complies with:

$$
\ddot{a}+\frac{2 g \sin (\theta)}{L_{w}} a+\frac{1}{L_{w} \rho_{1, \mathrm{ref}}}\left(P_{2,0}^{r}\left(\frac{b_{0}}{l_{0}-a}\right)^{\gamma_{2}}-P_{2,0}^{l}\left(\frac{a_{0}}{a}\right)^{\gamma_{2}}\right)=\frac{g l_{0} \sin (\theta)}{L_{w}} .
$$


A non-linear ordinary differential equation is obtained which may be solved numerically using a classical RungeKutta method. Once $a(t)$ is known, $b(t)$ is deduced from $b(t)=l_{0}-a(t)$, the air pressures are calculated from C.6 and the water velocity verifies $v_{1}=\dot{a}$.

Remark C.1 (Open pipe). When the pipe is open at the boundaries, the air pressure satisfies $P_{2}^{r}(t)=P_{2}^{l}(t)=$ $P_{\text {atm }}, \forall t$, so that (C.7) writes:

$$
\ddot{a}+\frac{2 g \sin (\theta)}{L_{w}} a=\frac{g l_{0} \sin (\theta)}{L_{w}} .
$$

An harmonic oscillator is obtained whose solution is:

$$
a(t)=a_{e q}+\left(a_{0}-a_{e q}\right) \cos \left(\omega_{0} t\right)
$$

where $a_{e q}=\frac{l_{0}}{2}$ is the equilibrium position and $\omega_{0}=\sqrt{\frac{2 g \sin (\theta)}{L_{w}}}$ is the pulsation of oscillations.

Remark C.2 (Oscillations around the equilibrium state). As detailed in [25], the solution of (C.7) may be linearized around its equilibrium state. Taking $a(t)$ under the form $a=a_{e q}+\delta$ where $a_{e q}$ denotes the equilibrium position and assuming $|\delta| \ll\left|a_{e q}\right|$, one obtains:

$$
\ddot{\delta}+\omega^{2} \delta=0,
$$

where:

$$
\omega^{2}=\omega_{0}^{2}+\omega_{a}^{2}, \omega_{0}^{2}=\frac{2 g \sin (\theta)}{L_{w}}, \omega_{a}^{2}=\frac{\gamma_{2}}{L_{w} \rho_{1, \text { ref }}}\left(\frac{P_{2, e q}^{l}}{a_{e q}}+\frac{P_{2, e q}^{r}}{b_{e q}}\right) .
$$

Thus, an harmonic oscillator is obtained whose pulsation features an additional contribution $\omega_{a}$ due to air pocket entrapment. Indeed, the pulsation $\omega_{0}$ is the corresponding pulsation when the pipe is open, see Remark C.1.

Remark C.3 (Influence of air pocket entrapment on oscillations). Considering $P_{2, e q}^{l} \sim P_{2, e q}^{r} \sim P_{2, d}, a_{e q} \sim$ $b_{e q} \sim d$ and $\theta=90^{\circ},(\mathrm{C} .11)$ yields:

$$
\frac{\omega}{\omega_{0}}=\sqrt{1+\frac{\gamma_{2} P_{2, d}}{\rho_{1, \mathrm{ref}} g d}} .
$$

The air entrapment has no influence on the pulsation of oscillations when $\omega \sim \omega_{0}$, which also writes $d \gg \frac{\gamma_{2} P_{2, d}}{\rho_{1, \text { ref }} g}$. With $P_{2, d} \sim 1$ bar, it yields $d \gg 15 \mathrm{~m}$. Therefore, the effects of air pocket entrapment are experienced in a closed pipe even if the boundaries are taken far from the liquid phase.

\section{REFERENCES}

[1] V.I. Agoshkov, D. Ambrosi, V. Pennati, A. Quarteroni, and F. Saleri. Mathematical and numerical modelling of shallow water flow. Computational Mechanics, 11(5):280-299, 1993.

[2] A. Ambroso, C. Chalons, F. Coquel, and T. Galié. Relaxation and numerical approximation of a two-fluid two-pressure diphasic model. ESAIM: Mathematical Modelling and Numerical Analysis, 43(6):1063-1097, 2009.

[3] A. Ambroso, C. Chalons, and P.-A. Raviart. A Godunov-type method for the seven-equation model of compressible two-phase flow. Computers \&5 Fluids, 54:67-91, 2012.

[4] K. Arai and K. Yamamoto. Transient analysis of mixed free- surface-pressurized flows with modified slot model (part 1: Computational model and experiment). Proc. FEDSM03 4th ASME-JSME Joint Fluids Engineering Conf., pages 2907-2913, 2003.

[5] F. Aureli, A. Dazzi, A. Maranzoni, and P. Mignosa. Validation of single- and two-equation models for transient mixed flows: a laboratory test case. Journal of Hydraulic Research, 53(4):440-451, 2015.

[6] M. R. Baer and J. W. Nunziato. A two phase mixture theory for the deflagration to detonation (DDT) transition in reactive granular materials. International Journal of Multiphase Flow, 12(6):861-889, 1986.

[7] A.J.C. Barré de Saint Venant. Théorie du mouvement non-permanent des eaux avec application aux crues des rivières et à l'introduction des marées dans leur lit. C.R. Acad. Sc. Paris., 73:147-154, 1871. 
[8] C. Berthon, B. Braconnier, and B. Nkonga. Numerical approximation of a degenerate non-conservative multifluid model: relaxation scheme. International Journal for Numerical Methods in Fluids, 48:85-90, 2005.

[9] F. Bouchut. Nonlinear stability of finite volume methods for hyperbolic conservation laws, and well-balanced schemes for sources. Birkhauser, 2004.

[10] C. Bourdarias, M. Ersoy, and S. Gerbi. Unsteady flows in non uniform closed water pipes: a full kinetic approach. Numerische Mathematik, 128(2):217-263, 2014.

[11] C. Bourdarias and S. Gerbi. A finite volume scheme for a model coupling free surface and pressurised flows in pipes. Journal of Computational and Applied Mathematics, 209:1-47, 2007.

[12] S. Bousso, M. Daynou, and M. Fuamba. Numerical modeling of mixed flows in storm water systems: Critical review of literature. Journal of Hydraulic Engineering, 139(4):385-396, 2013.

[13] R.P. Brent. An algorithm with guaranteed convergence for finding a zero of a function. Computer Journal, 14(4):422-425, 1971.

[14] H. Capart, X. Sillen, and Y. Zech. Numerical and experimental water transients in sewer pipes. Journal of Hydraulic Research, 35(5):659-672, 1997.

[15] J.A. Cardle, C.C.S. Song, and M. Yuan. Measures of mixed transient flows. Journal of Hydraulic Engineering, 115(2):169-182, 1989.

[16] C. Chalons, F. Coquel, S. Kokh, and N. Spillane. Large time-step numerical scheme for the seven-equation model of compressible two-phase flows. Springer Proceedings in Mathematics, 4:225-233, 2011.

[17] C. Chalons, M. Girardin, and S. Kokh. Large time-step and asymptotic preserving numerical schemes for the gas dynamics equations with source terms. SIAM Journal on Scientific Computing, 35(6):a2874-a2902, 2013.

[18] F. Coquel, K. El Amine, E. Godlewski, B. Perthame, and P. Rascle. A numerical method using upwind schemes for the resolution of two-phase flows. Journal of Computational Physics, 136(2):272-288, 1997.

[19] F. Coquel, T. Gallouët, J.-M. Hérard, and N. Seguin. Closure laws for a two-fluid two-pressure model. C. R. Acad. Sci. Paris, 334(I):927-932, 2002.

[20] F. Coquel, E. Godlewski, and N. Seguin. Relaxation of fluid systems. Mathematical Models and Methods in Applied Sciences, $22(8), 2012$.

[21] F. Coquel, J.-M. Hérard, and K. Saleh. A splitting method for the isentropic Baer-Nunziato two-phase flow model. ESAIM: Proceedings, 38(3):241-256, 2012.

[22] F. Coquel, J.-M. Hérard, and K. Saleh. A positive and entropy-satisfying finite volume scheme for the Baer-Nunziato model. Journal of Computational Physics, 330:401-435, 2017.

[23] F. Coquel, J.-M. Hérard, K. Saleh, and N. Seguin. A robust entropy-satisfying finite volume scheme for the isentropic BaerNunziato model. ESAIM: Mathematical Modelling and Numerical Analysis, 48:165-206, 2013.

[24] J.-A. Cunge and M. Wegner. Numerical integration of barré de Saint-Venant's flow equations by means of an implicit scheme of finite differences. La Houille Blanche, 1:33-39, 1964.

[25] C. Demay. Modelling and simulation of transient air-water two-phase flows in hydraulic pipes. PhD thesis, Université Savoie Mont Blanc, 2017. URL: https://tel.archives-ouvertes.fr/tel-01651078.

[26] C. Demay, C. Bourdarias, B. de Laage de Meux, S. Gerbi, and J.-M. Hérard. Numerical simulation of a compressible twolayer model: a first attempt with an implicit-explicit splitting scheme. Journal of Computational and Applied Mathematics, 346:357-377, 2019.

[27] C. Demay and J.-M. Hérard. A compressible two-layer model for transient gas-liquid flows in pipes. Continuum Mechanics and Thermodynamics, 29(2):385-410, 2017.

[28] G. Dimarco, R. Loubère, and M.-H. Vignal. Study of a new asymptotic preserving scheme for the euler system in the low Mach number limit. SIAM Journal on Scientific Computing, 39(5):A2099-A2128, 2017.

[29] M. Ferrari, A. Bonzanini, and P. Poesio. A 5-equation, transient, hyperbolic, 1-dimensional model for slug capturing in pipes. International Journal for Numerical Methods in Fluids, 85(6):327-362, 2017.

[30] T. Gallouët, J.-M. Hérard, and N. Seguin. Numerical modeling of two-phase flows using the two-fluid two-pressure approach. Mathematical Models and Methods in Applied Sciences, 14(05):663-700, 2004.

[31] S.L. Gavrilyuk. The structure of pressure relaxation terms : one-velocity case. EDF report H-I83-2014-00276-EN, 2014.

[32] J.-F. Gerbeau and B. Perthame. Derivation of viscous Saint-Venant system for laminar shallow water; numerical validation. Discrete Contin. Dyn. Syst. Ser. B, 1:89-102, 2001.

[33] E. Godlewski, M. Parisot, J. Sainte-Marie, and F. Wahl. Congested shallow water model: roof modelling in free surface flow. ESAIM: Mathematical Modelling and Numerical Analysis, 2018.

[34] E. Godlewski and P.-A. Raviart. Numerical approximation of hyperbolic systems of conservation laws. Springer New York, 1996.

[35] J.-M. Hérard and O. Hurisse. A fractional step method to compute a class of compressible gas-liquid flows. Computers 6 Fluids, 55:57-69, 2012.

[36] D. Iampietro, F. Daude, P. Galon, and J.-M. Hrard. A mach-sensitive implicitexplicit scheme adapted to compressible multiscale flows. Journal of Computational and Applied Mathematics, 340:122 - 150, 2018. 
[37] R.I. Issa and M.H.W. Kempf. Simulation of slug flow in horizontal and nearly horizontal pipes with the two-fluid model. International Journal of Multiphase Flow, 29:69-95, 2003.

[38] F. Kerger, P. Archambeau, S. Erpicum, B.J. Dewals, and M. Pirotton. An exact Riemann solver and a Godunov scheme for simulating highly transient mixed flows. Journal of Computational and Applied Mathematics, 235(8):2030-2040, 2011.

[39] D. Lannes. On the dynamics of floating structures. Annals of PDE, 3(1):11, 2017.

[40] A.S. Léon, M.S. Ghidaoui, A.R. Schmidt, and M.H. Garcia. A robust two-equation model for transient-mixed flows. Journal of Hydraulic Research, 48(1):44-56, 2010.

[41] A. Line and J. Fabre. Stratified gas liquid flow. Encyclopedia of Heat Transfer, pages 1097-1101, 1997.

[42] H. Lochon, F. Daude, P. Galon, and J.-M. Hérard. HLLC-type Riemann solver with approximated two-phase contact for the computation of the Baer-Nunziato two-fluid model. Journal of Computational Physics, 326:733-762, 2016.

[43] L. Ramezani, B. Karney, and A. Malekpour. Encouraging effective air management in water pipelines: A critical review. Journal of Water Resources Planning and Management, 142(12), 2016.

[44] V. H. Ransom and D. L. Hicks. Hyperbolic two-pressure models for two-phase flow. Journal of Computational Physics, 53:124$151,1984$.

[45] J. Schütz and S. Noelle. Flux splitting for stiff equations: A notion on stability. SIAM Journal on Scientific Computing, 64:522-540, 2015.

[46] V.L. Streeter, E.B. Wylie, and K.W. Bedford. Fluid Mechanics. McGraw-Hill, 1998.

[47] Y. Taitel and A.E. Dukler. A model for predicting flow regime transitions in horizontal and near horizontal gas-liquid flow. AIChE J., 22:47-55, 1976.

[48] S.-A. Tokareva and E.-F. Toro. HLLC-type Riemann solver for the Baer-Nunziato equations of compressible two-phase flow. Journal of Computational Physics, 229(10):3573-3604, 2010.

[49] B.C. Trindade and J.G. Vasconcelos. Modeling of water pipeline filling events accounting for air phase interactions. Journal of Hydraulic Engineering, 139(9):921-934, 2013.

[50] J.G. Vasconcelos, S.J. Wright, and P.L. Roe. Improved simulation of flow regime transition in sewers: Two-component pressure approach. Journal of Hydraulic Engineering, 132(6):553-562, 2006.

[51] J.G. Vasconcelos, S.J. Wright, and P.L. Roe. Numerical oscillations in pipe-filling bore predictions by shock-capturing models. Journal of Hydraulic Engineering, 135(4):296-305, 2009.

[52] H. Zakerzadeh and S. Noelle. A note on the stability of implicit-explicit flux splittings for stiff hyperbolic systems. IGPM report 449, 2016. 